\title{
Multiplicative Square Functions
}

\author{
María José González and Artur Nicolau
}

\begin{abstract}
We study regularity properties of a positive measure in the euclidean space in terms of two square functions which are the multiplicative analogues of the usual martingale square function and of the Lusin area function of a harmonic function. The size of these square functions is related to the rate at which the measure doubles at small scales and determines several regularity properties of the measure. We consider the non-tangential maximal function of the logarithm of the densities of the measure in the dyadic setting, and of the logarithm of the harmonic extension of the measure, in the continuous setting. We relate the size of these maximal functions to the size of the corresponding square functions. Fatou type results, $L^{p}$ estimates and versions of the Law of the Iterated Logarithm are proved. As applications we introduce a hyperbolic version of the Lusin Area function of an analytic mapping from the unit disc into itself, and use it to characterize inner functions. Another application to the theory of quasiconformal mappings is given showing that our methods can also be applied to prove a result by Din'kyn's on the smoothness of quasiconformal mappings of the disc.
\end{abstract}

\section{Introduction}

A positive measure $\mu$ in $\mathbb{R}^{N}$ is called doubling if there exists a positive constant $C=C(\mu)$ such that

$$
\mu(2 Q) \leq C \mu(Q)
$$

for any cube $Q \subset \mathbb{R}^{N}$, where $2 Q$ denotes the cube which the same center as $Q$ and twice the sidelength. Lebesgue measure $d m$ is doubling and, as a

2000 Mathematics Subject Classification: 42B25, 31A20.

Keywords: Doubling measure, martingale, positive harmonic functions, square functions, hyperbolic derivative, quasiconformal mappings. 
matter of fact, it is the only doubling measure for which the constant $C$ can be taken $C=2^{N}$. However, classical constructions $([7,13])$ provide examples of doubling measures which are singular with respect to Lebesgue measure. See also [2], [12], [24], [33].

Our main purpose is to describe regularity properties of a measure, such as being singular, in terms of two different square functions. The first square function is related to the rate at which the measure doubles at small scales, while the second one is connected to the logarithm of the harmonic extension of the measure. An important result in this direction, stated below in a different way, was proved by L. Carleson ([13]) for $N=1$, when studying regularity properties of quasiconformal mappings. See also [27, p. 5], and [16] for the extension to $\mathbb{R}^{N}$.

Theorem A. (a) Let $\mu$ be a doubling measure in $\mathbb{R}^{N}$. Consider

$$
w(t)=\sup \left|\frac{\mu(Q) 2^{N}}{\mu(2 Q)}-1\right|
$$

where the supremum is taken over all cubes $Q$ in $\mathbb{R}^{N}$ of sidelength smaller than t. Assume

$$
\int_{0} w^{2}(t) \frac{d t}{t}<\infty
$$

Then, the measure $\mu$ is absolutely continuous and its density $f$ satisfies

$$
\int_{Q} \exp \left(C f^{2}\right) d m<\infty,
$$

for any cube $Q \subset \mathbb{R}^{N}$ and any $C>0$.

(b) Let $w:[0,1] \rightarrow[0, \infty]$ be an increasing function with $w(0)=0$. Assume that for some $0<\varepsilon<1$, the function $w(t) / t^{1-\varepsilon}$ is non-decreasing. Assume also

$$
\int_{0} w^{2}(t) \frac{d t}{t}=\infty
$$

Then, there exists a singular doubling measure $\mu$ on $\mathbb{R}^{N}$ such that

$$
\left|\frac{\mu(Q) 2^{N}}{\mu(2 Q)}-1\right| \leq w(l(Q)),
$$

for any cube $Q \subset \mathbb{R}^{N}$.

So, the quadratic condition

$$
\int_{0} w^{2}(t) \frac{d t}{t}=\infty
$$

governs the existence of singular measures that double at rate $w(t)$. However, this is a global result because the function $w(t)$ controls the error done by the measure when doubling at all cubes of sidelength $t$. 
We are interested in local versions of this result. In the additive setting, local results of this sort were proved by Stein and Zygmund [35, p. 262]. They showed that a real valued function $f$ on $\mathbb{R}^{N}$ has an ordinary derivative at almost every point of a set $E$ if and only if both

$$
\begin{gathered}
f(x+t)+f(x-t)-2 f(x)=O(|t|), \quad t \rightarrow 0, \\
\int_{|t| \leq \delta}|f(x+t)+f(x-t)-2 f(x)|^{2} \frac{d t}{|t|^{N+2}}<\infty
\end{gathered}
$$

for almost every $x \in E$.

Both our results and methods are better explained in the dyadic setting. Let $Q_{0}$ be the unit cube in $\mathbb{R}^{N}$. Let us consider the dyadic decomposition of $Q_{0}$, that is, for $k=1,2, \ldots$, we consider the collection $F_{k}$ of the $2^{k N}$ pairwise disjoint subcubes of $Q_{0}$ of the form

$$
Q=\left[j_{1} 2^{-k},\left(j_{1}+1\right) 2^{-k}\right) \times \cdots \times\left[j_{N} 2^{-k},\left(j_{N}+1\right) 2^{-k}\right),
$$

where $j_{1}, \ldots, j_{N}$ are integers between 0 and $2^{k}-1$. So

$$
m(Q)=|Q|=2^{-k N}
$$

A positive measure $\mu$ on $Q_{0}$ is called dyadic doubling if there exists a constant $C=C(\mu)>0$ such that

$$
\mu(\widetilde{Q}) \leq C \mu(Q)
$$

for any dyadic subcube $Q$ of $Q_{0}$, where $\widetilde{Q}$ is the smallest dyadic subcube of $Q_{0}$ which properly contains $Q$. These measures were completely characterized in terms of their Haar coefficients by Fefferman, Kenig and Pipher ([17]). Results on null sets of (dyadic) doubling measures can be found in [25], [39], [40].

A dyadic martingale on $Q_{0}$ is a sequence of functions $\left\{S_{n}\right\}$ from $Q_{0}$ to $\mathbb{R}$ such that $S_{n}$ is $F_{n}$-measurable and the conditional expectation of $S_{n+1}$ on $F_{n}$ is $S_{n}, n=0,1,2, \ldots$ These two conditions may be rephrased as: $S_{n}$ is constant (which we denote $S_{n}(\widetilde{Q})$ ) in each cube $\widetilde{Q}$ of $F_{n}$ and

$$
S_{n}(\widetilde{Q})=\frac{1}{2^{N}} \sum S_{n+1}(Q),
$$

where the sum is taken over all cubes $Q$ of $F_{n+1}$ contained in $\widetilde{Q}$. Note that $S_{n}(x)$ represents the value of the martingale on the cube $Q_{n}(x)$, where $Q_{n}(x)$ denotes the unique dyadic cube of sidelenght $2^{-n}$ containing $x$. 
The (truncated) maximal function of the martingale $\left\{S_{n}\right\}$ is

$$
S_{m}^{*}(x)=\sup _{0 \leq n \leq m}\left|S_{n}(x)\right|, \quad m=0,1,2, \ldots
$$

If $m=\infty$, we simply write $S^{*}(x)$. The (truncated) martingale square function of $\left\{S_{n}\right\}$ is defined as

$$
\langle S\rangle_{m}(x)=\left(\sum_{k=1}^{m}\left\|\left(S_{k}-S_{k-1}\right) \chi_{Q_{k-1}(x)}\right\|_{\infty}^{2}\right)^{1 / 2}, \quad x \in Q_{0} .
$$

In dimension one, this is the more traditional square function

$$
\langle S\rangle_{m}(x)=\left(\sum_{k=1}^{m}\left(S_{k}(x)-S_{k-1}(x)\right)^{2}\right)^{1 / 2} .
$$

If $m=\infty$, we simply write $\langle S\rangle(x)$. It is well known that many properties of the martingale $\left\{S_{n}\right\}$ can be described using its square function. Burckholder and Gundy proved that the sets

$$
\left\{x \in Q_{0}: \lim _{n \rightarrow \infty} S_{n}(x) \text { exists }\right\} \quad \text { and } \quad\left\{x \in Q_{0}:\langle S\rangle(x)<\infty\right\}
$$

can only differ on a set of Lebesgue measure 0 . Also, for $0<p<\infty$, the maximal function

$$
S^{*}(x)=\sup _{n}\left|S_{n}(x)\right|
$$

is in $L^{p}\left(Q_{0}\right)$ if and only if $\langle S\rangle \in L^{p}\left(Q_{0}\right)$. See [9] and [10]. These results give comparisons between $S^{*}$ and $\langle S\rangle$ on the set where they are finite. In its complement, the following Law of the Iterated Logarithm holds:

$$
\limsup _{n \rightarrow \infty} \frac{\left|S_{n}(x)\right|}{\langle S\rangle_{n}(x) \sqrt{2 \log \log \langle S\rangle_{n}(x)}} \leq 1,
$$

at almost every point $x \in\left\{x \in Q_{0}: \sup _{n}\left|S_{n}(x)\right|=\infty\right\}$ ([35, 6]). An important result in this direction is the following good- $\lambda$ inequality established by Chang, Wilson and Wolff,

$$
\begin{aligned}
\mid\left\{x \in Q_{0}:\right. & \left.S^{*}(x)>2 \lambda, \quad\langle S\rangle(x)<\varepsilon \lambda\right\} \mid \leq \\
& \leq C \exp \left(-\frac{1}{2}\left(\frac{1-\varepsilon}{\varepsilon}\right)^{2}\right)\left|\left\{x \in Q_{0}: S^{*}(x)>\lambda\right\}\right| .
\end{aligned}
$$

Here $\varepsilon$ is any number in $(0,1)$ and $C$ is a universal constant $([14])$. 
Given a positive measure $\mu$ on $Q_{0}$, one may consider the dyadic martingale

$$
S_{k}=\sum_{Q \in F_{k}} \frac{\mu(Q)}{|Q|} \chi_{Q}, \quad k=1,2, \ldots
$$

Thus, many properties of the martingale $\left\{S_{k}\right\}$ and hence of the measure $\mu$, can be described in terms of its quadratic variation,

$$
\langle S\rangle^{2}(x)=\sum_{k=1}^{\infty} \max \left\{\frac{\mu(Q)}{|Q|}-\frac{\mu\left(Q_{k-1}(x)\right)}{\left|Q_{k-1}(x)\right|}\right\}^{2},
$$

where the maximum is taken over all cubes $Q \in F_{k}$ contained in $Q_{k-1}(x)$. For instance, it is easy to show that, for $1<p<\infty, \mu$ has a density (with respect to Lebesgue measure) in $L^{p}\left(Q_{0}\right)$ if and only if the square function of the martingale is in $L^{p}\left(Q_{0}\right)$. Because of the additive nature of these expressions, the martingale $\left\{S_{k}\right\}$ is very well suited to study additive properties of the measure $\mu$. See [2], [16], [27], [26]. When dealing with multiplicative properties of a measure, such as doubling conditions, it is natural to consider, somehow, the logarithm of $S_{n}$. This point of view has already been used by other authors. For instance, we mention the work of Fefferman, Kenig and Phiper [17] in connection with the theory of weights (see also [11], [37]) and the work of P. MacManus ([29]) on quasiconformal mappings.

Next we introduce a square function of a positive measure which is, in some sense, a multiplicative analogue of the square function which arises from the martingale. Given a dyadic doubling measure $\mu$ on $Q_{0}$, we consider the dyadic square function

$$
A^{2}(\mu)(x)=\sum_{k=1}^{\infty} d_{k}^{2}(x), \quad x \in Q_{0}
$$

where

$$
d_{k}(x)=\max \left\{\left|1-\frac{2^{N} \mu(Q)}{\mu\left(Q_{k-1}(x)\right)}\right|: Q \in F_{k}, Q \subset Q_{k-1}(x)\right\} .
$$

So, the function $A^{2}(\mu)(x)$ measures the error done by $\mu$ when doubling on all dyadic cubes containing $x \in Q_{0}$. In dimension 1 , this is

$$
A^{2}(\mu)(x)=\sum_{k=1}^{\infty}\left(1-\frac{2 \mu\left(I_{k}(x)\right)}{\mu\left(I_{k-1}(x)\right)}\right)^{2},
$$

where $I_{j}(x), j=1,2, \ldots$, is the unique dyadic interval of length $2^{-j}$ which contains $x \in I_{0}$. Our first result is the following. 
Theorem 1.1. Let $\mu$ be a dyadic doubling measure in $Q_{0}$ and $A^{2}(\mu)$ its dyadic square function. Let $Q_{n}(x)$ be the unique dyadic cube in $F_{n}$ which contains $x \in Q_{0}$. Then,

(a) The sets

$$
\left\{x \in Q_{0}: \lim _{n \rightarrow \infty} \frac{\mu\left(Q_{n}(x)\right)}{\left|Q_{n}(x)\right|}>0\right\} \quad \text { and } \quad\left\{x \in Q_{0}: A^{2}(\mu)(x)<\infty\right\}
$$

can only differ in a set of Lebesgue measure 0.

(b) Consider the maximal function

$$
M(\log \mu)(x)=\sup _{n}\left|\log \frac{\mu\left(Q_{n}(x)\right)}{\left|Q_{n}(x)\right|}\right|, \quad x \in Q_{0} \quad .
$$

Then, for $0<p<\infty, M(\log \mu) \in L^{p}\left(Q_{0}\right)$ if and only if $A^{2}(\mu) \in L^{p}\left(Q_{0}\right)$.

(c) Let $f$ be the density of $\mu$ respect Lebesgue measure. Then, for $1<p<\infty$, $\log f \in L^{p}\left(Q_{0}\right)$ if and only if $A^{2}(\mu) \in L^{p}\left(Q_{0}\right)$.

(d) There exists a constant $C=C(N)$, only depending on the dimension, such that $\exp \left(C A^{2}(\mu)\right) \in L^{1}\left(Q_{0}\right)$ implies that $\mu$ and Lebesgue measure are mutually absolutely continuous.

(e) There exists a constant $c=c(N)$, only depending on the dimension, such that if $A^{2}(\mu)(x)<1$ for any $x \in Q_{0}$, then $\exp \left(c M^{2}(\log \mu)\right) \in L^{1}\left(Q_{0}\right)$.

The statements in (a), (b) and (c) are the analogues, in our setting, of the classical results by Burkholder and Gundy cited above, while (d) and (e) are consequences of certain good- $\lambda$ inequalities relating the growth of $M(\log u)$ and $A^{2}(\mu)$ and which are the analogues of the subgaussian estimates of Chang, Wilson and Wolff. As a consequence of (a) we obtain

Corollary 1.2. Let $\mu$ be a dyadic doubling measure in $Q_{0}$ and let $A^{2}(\mu)$ be its dyadic square function. Then, $\mu$ is singular with respect to Lebesgue measure if and only if

$$
A^{2}(\mu)(x)=\infty
$$

at almost $(d m)$ every point $x \in Q_{0}$.

The main idea in the proof is to consider the logarithmic transformation of the martingale $\left\{S_{n}\right\}$. More concretely, given a dyadic doubling measure $\mu$ on $Q_{0}$, we consider the dyadic martingale

$$
S_{n}=\sum_{Q \in F_{n}} \frac{\mu(Q)}{|Q|} \chi_{Q}
$$


It is easy to see that there exists a unique (except for adding constants) process $X_{n}$ verifying the following two conditions:

(a) $X_{n}$ is $F_{n-1}$-measurable, that is, $X_{n}$ is constant on any cube of $F_{n-1}$,

(b) $T_{n}=\log S_{n}+X_{n}$ is a dyadic martingale.

It turns out that the martingale $\left\{T_{n}\right\}$ is the inverse of the exponential transformation of $\left\{S_{n}\right\}$ (see [27, p. 27], [6, p. 47]). Actually the process $X_{n}$ can be given explicitly by

$$
X_{n}=\sum_{Q \in F_{n}} X_{n}(Q) \chi_{Q}
$$

where

$$
X_{n}(Q)=-\sum \log \frac{\mathrm{g} \cdot \mathrm{m} \cdot\left(Q_{j}\right)}{\operatorname{a} \cdot \mathrm{m} \cdot\left(Q_{j}\right)}
$$

Here the sum is taken over all dyadic cubes $\left\{Q_{j}\right\}$ which properly contain $Q$. Also, if $Q \in F_{l}$ and $Q_{1}, \ldots, Q_{2^{N}}$ are the dyadic cubes in $F_{l+1}$ contained in $Q$, then g.m. $(Q)$ (a.m. $(Q)$ ) denotes the geometric (arithmetic) mean of the density of $\mu$ over $Q_{1}, \ldots, Q_{2^{N}}$, that is

$$
\text { g.m. }(Q)=\prod_{i=1}^{2^{N}}\left(\frac{\mu\left(Q_{i}\right)}{\left|Q_{i}\right|}\right)^{1 / 2^{N}}, \quad \text { a.m. }(Q)=\frac{\mu(Q)}{|Q|}=\frac{1}{2^{N}} \sum_{i=1}^{2^{N}} \frac{\mu\left(Q_{i}\right)}{\left|Q_{i}\right|} \text {. }
$$

Now, the main observations are that, under the doubling hypothesis, the quadratic variation $\langle T\rangle^{2}(x)$ is pointwise comparable to $A^{2}(\mu)(x)$, and $X_{n}(x)$ is pointwise comparable to the truncated area function $A_{n}^{2}(\mu)(x)$, that is

$$
X_{n}(x) \simeq \sum_{k=1}^{n} d_{k}^{2}(x)=A_{n}^{2}(\mu)(x) .
$$

Let

$$
T^{*}(x)=\sup _{n}\left|T_{n}(x)\right|=\sup _{n}\left|\log \frac{\mu\left(Q_{n}(x)\right)}{\left|Q_{n}(x)\right|}+X_{n}(x)\right| .
$$

Now, since $\langle T\rangle$ is comparable to $A(\mu)$, the usual stopping time arguments which go back to Burkholder and Gundy (see $[9,10]$ ) used to compare the distribution functions of $T^{*}(x)$ and $\langle T\rangle(x)$ can be used to compare $M(\log \mu)$ and $A^{2}(\mu)$ and obtain (a) and (b) in Theorem 1.1. However the proof of (d) and (e) uses the deeper subgaussian estimates proved by Chang, Wilson and Wolff mentioned above ([14]). These subgaussian estimates are also very closely related to the law of the iterated logarithm. In our case we get that the logarithm of the density of $\mu$ behaves very closely to $X_{n}(x)$ on the set $\left\{x \in Q_{0}: \lim _{n \rightarrow \infty} \frac{\mu\left(Q_{n}(x)\right)}{\left|Q_{n}(x)\right|}=0\right\}$, which coincides, except for a set of Lebesgue measure 0, with $\left\{x \in Q_{0}: X(x)=\infty\right\}=\left\{x \in Q_{0}: A(\mu)(x)=\infty\right\}$. 
Theorem 1.3. Let $\mu$ be a dyadic doubling measure on $Q_{0}$ and let $X_{n}$ be given by (1.2). Then there exists a constant $C$ depending on the dimension and on the doubling constant of $\mu$, such that at almost every point $x \in\{x \in$ $\left.Q_{0}: \lim _{n \rightarrow \infty} \frac{\mu\left(Q_{n}(x)\right)}{\left|Q_{n}(x)\right|}=0\right\}$, one has

$$
C^{-1} \leq \limsup _{n \rightarrow \infty} \frac{\left|\log \frac{\mu\left(Q_{n}(x)\right)}{\left|Q_{n}(x)\right|}+X_{n}(x)\right|}{\sqrt{X_{n}(x) \log \log X_{n}(x)}} \leq C .
$$

Since, as mentioned before, $X_{n}(x)$ is comparable to $A_{n}^{2}(\mu)(x)$, we deduce Corollary 1.4. Let $\mu$ be a dyadic doubling measure on $Q_{0}$, let $A^{2}(\mu)$ be its square function and let $X_{n}$ be given by (1.2). Then at almost every point $x \in\left\{x \in Q_{0}: \lim _{n \rightarrow \infty} \frac{\mu\left(Q_{n}(x)\right)}{\left|Q_{n}(x)\right|}=0\right\}$,

$$
\lim _{n \rightarrow \infty} \frac{\left|\log \frac{\mu\left(Q_{n}(x)\right)}{\left|Q_{n}(x)\right|}\right|}{X_{n}(x)}=1 .
$$

Also, there exists a constant $C$ depending on the dimension and on the doubling constant of $\mu$ such that

$$
C^{-1}<\frac{\left|\log \frac{\mu\left(Q_{n}(x)\right)}{\left|Q_{n}(x)\right|}\right|}{A_{n}^{2}(\mu)(x)}<C,
$$

at almost every point $x \in\left\{x \in Q_{0}: \lim _{n \rightarrow \infty} \frac{\mu\left(Q_{n}(x)\right)}{\left|Q_{n}(x)\right|}=0\right\}$, for $n$ sufficiently large.

It should be observed that

$$
\log \frac{\mu\left(Q_{n}(x)\right)}{\left|Q_{n}(x)\right|} \leq C(\mu) \sum_{k=1}^{n} d_{k}(x)
$$

is the best possible pointwise estimate. However, the last result shows that this inequality can be substantially improved at almost every point on the set $\left\{x \in Q_{0}: \lim _{n \rightarrow \infty} \frac{\mu\left(Q_{n}(x)\right)}{\left|Q_{n}(x)\right|}=0\right\}$.

The second part of our work is devoted to study the relation between singularity, as well as other regularity properties of a measure, and an square function which arises from the harmonic extension of the measure. Let $u$ be a harmonic function in the upper-half space $\mathbb{R}_{+}^{N+1}=\left\{(x, y): x \in \mathbb{R}^{N}, y>0\right\}$. Given $\alpha>0$ and $x_{0} \in \mathbb{R}^{N}$, we denote by $\Gamma\left(x_{0}\right)=\Gamma_{\alpha}\left(x_{0}\right)$ the (truncated) cone with vertex at $x_{0}$ of aperture $\alpha$, that is

$$
\Gamma_{\alpha}\left(x_{0}\right)=\left\{(x, y) \in \mathbb{R}_{+}^{N+1}:\left|x-x_{0}\right|<\alpha y, 0<y<1\right\} .
$$


Given $t \geq 0$, the (doubly) truncated cone $\Gamma_{\alpha}\left(x_{0}, t\right)$ with vertex at $x_{0} \in \mathbb{R}^{N}$ is

$$
\Gamma_{\alpha}\left(x_{0}, t\right)=\left\{(x, y) \in \mathbb{R}_{+}^{N+1}:\left|x-x_{0}\right|<\alpha y, t<y<1\right\}
$$

and the (doubly) truncated maximal function of $u$ is

$$
M_{\alpha}(u)\left(x_{0}, t\right)=\sup \left\{\mid u(x, y):(x, y) \in \Gamma_{\alpha}\left(x_{0}, t\right)\right\}
$$

and when $t=0$, we simply write $M_{\alpha}(u)\left(x_{0}\right)$.

The (doubly) truncated area function of $u$ is defined by

$$
A_{\alpha}^{2}(u)\left(x_{0}, t\right)=\int_{\Gamma_{\alpha}\left(x_{0}, t\right)}|\nabla u(x, y)|^{2} y^{1-N} d x d y
$$

and when $t=0$, we simply write $A_{\alpha}^{2}(u)\left(x_{0}\right)$.

The size of the area function and the non tangential maximal function are intimately related. For instance, a series of results by Marcinkiewicz, Zygmund, Spencer, Calderón and Stein assert that for any $\alpha>0$, the following two sets

$$
\left\{x \in \mathbb{R}^{N}: u \text { has non-tangential limit at } x\right\}, \quad\left\{x \in \mathbb{R}^{N}: A_{\alpha}(u)(x)<\infty\right\},
$$

can only differ in a set of Lebesgue measure 0. Also, C. Fefferman and E. Stein $([18])$ proved that for any $0<p<\infty$, there exists a constant $C=C(p, N)>0$ such that for any harmonic function $u$ on $\mathbb{R}_{+}^{N+1}$ with $\lim _{t \rightarrow \infty} u(x, t)=0$, one has

$$
C^{-1}\left\|A_{\alpha}(u)\right\|_{p} \leq\left\|M_{\alpha}(u)\right\|_{p} \leq C\left\|A_{\alpha}(u)\right\|_{p} .
$$

So that, the distribution functions of $M(u)$ and $A(u)$ have, roughly speaking, the same rate of growth. Many authors have proved good $-\lambda$ inequalities for $M(u)$ and $A(u)$, which are direct comparisons of their distribution functions ([10, 18]). Muray and Uchiyama ([31]) proved that given $0<\beta<\alpha$, there exists a constant $K>1$ and positive numbers $C_{1}, C_{2}$ such that for any $0<\varepsilon<1$ and any $\lambda>0$ one has

$$
\begin{aligned}
\mid\left\{x \in \mathbb{R}^{N}:\right. & \left.A_{\beta}(u)(x)>K \lambda, M_{\alpha}(u)<\varepsilon \lambda\right\} \mid \\
& \leq C_{1} e^{-C_{2} / \varepsilon^{2}}\left|\left\{x \in \mathbb{R}^{N}: A_{\beta}(u)(x)>\lambda\right\}\right|
\end{aligned}
$$

and

$$
\begin{aligned}
\mid\left\{x \in \mathbb{R}^{N}:\right. & \left.M_{\beta}(u)(x)>K \lambda, A_{\alpha}(u)(x)<\varepsilon \lambda\right\} \mid \\
& \leq C_{1} e^{-C_{2} / \varepsilon}\left|\left\{x \in \mathbb{R}^{N}: M_{\beta}(u)(x)>\lambda\right\}\right| .
\end{aligned}
$$

Here $C_{1}, C_{2}$ are two absolute constants only depending on the dimension $N$ and on $\alpha, \beta$. 
The proof followed the traditional method of using Green's formula on sawtooth regions. Notice, however, that the second inequality gives a worst decay than the first one. Bañuelos, Klemes and Moore ([3]) improved this estimate and proved the subgaussian estimate

$$
\begin{aligned}
\mid\left\{x \in \mathbb{R}^{N}:\right. & \left.M_{\beta}(u)(x)>K \lambda, A_{\alpha}(u)(x)<\varepsilon \lambda\right\} \mid \\
& \leq C_{3} e^{-C_{4} / \varepsilon^{2}}\left|\left\{x \in \mathbb{R}^{N}: M_{\beta}(u)(x)>\lambda\right\}\right| .
\end{aligned}
$$

Their proof was based on reducing the estimate to an analogous estimate in the dyadic-martingale setting. This scheme was first used by Chang, Wilson and Wolff in ([14]).

These results give comparisons between $A_{\alpha}(u)$ and $M_{\beta}(u)$ on the set where these functions are finite. In its complement, Bañuelos, Klemes and Moore ([3]) proved a law of the iterated logarithm. This may be stated as follows: given $0<\beta<\alpha$ and $0<\gamma<1$, there exists a constant $C=C(\alpha, \beta, \gamma, N)>0$ such that for any harmonic function $u$ on $\mathbb{R}_{+}^{N+1}$, one has

$$
\limsup _{\substack{(y, t) \rightarrow(x, 0) \\(y, t) \in \Gamma_{\beta}(x)}} \frac{|u(y, t)|}{\sqrt{A_{\alpha}^{2}(u)(x, \gamma t) \log \log A_{\alpha}(u)(x, \gamma t)}} \leq C,
$$

for almost every $x \in\left\{x \in \mathbb{R}^{N}: A_{\alpha}(u)(x)=\infty\right\}$. The opposite inequality also holds if certain regularity properties of the function $u$ are required ([4]). It is especially interesting to us that if $u$ satisfies the Bloch condition

$$
y|\nabla u(x, y)| \leq C_{1}, \quad(x, y) \in \mathbb{R}_{+}^{N+1},
$$

for some fixed constant $C_{1}$, then

$$
\limsup _{\substack{(y, t) \rightarrow(x, 0) \\(y, t) \in \Gamma_{\alpha}(x)}} \frac{|u(y, t)|}{\sqrt{A_{\alpha}^{2}(u)(x, t) \log \log A_{\alpha}^{2}(u)(x, t)}} \geq C_{2},
$$

for almost every $x \in\left\{x \in \mathbb{R}^{N}: A_{\alpha}(u)(x)=\infty\right\}$.

Given a positive measure $\mu$ on $Q_{0}$, let $u$ be its harmonic extension. As in the martingale setting, the results mentioned above are very well suited to treat additive properties of the measure $\mu$. In our situation it is more natural to consider the function $\log u$ and its area function

$$
A_{\alpha}^{2}(\log u)(x)=\int_{\Gamma_{\alpha}(x)} \frac{|\nabla u(w, y)|^{2}}{u(w, y)^{2}} y^{1-N} d w d y, \quad x \in \mathbb{R}^{N} .
$$

This square function was first considered by J. Brossard [8], who gave a probabilistic proof of part (a) in the following result. 
Theorem 1.5. Let $\mu$ be a positive measure on $\mathbb{R}^{N}$ such that

$$
\int_{\mathbb{R}^{N}} \frac{d \mu(x)}{1+|x|^{N+1}}<\infty .
$$

Let $f$ be the density of $\mu$ with respect to Lebesgue measure. Fix $\alpha>0$. Let $u$ be the harmonic extension of $\mu$ and $A_{\alpha}(\log u)(x)$ its area function. Then

(a) The sets

$$
\left\{x \in \mathbb{R}^{N}: f(x)>0\right\} \quad \text { and } \quad\left\{x \in \mathbb{R}^{N}: A_{\alpha}(\log u)(x)<\infty\right\}
$$

can only differ in a set of Lebesgue measure 0.

(b) Consider the maximal function

$$
M_{\alpha}(\log u)(x)=\sup \left\{|\log u(w, y)|:(w, y) \in \Gamma_{\alpha}(x)\right\} .
$$

Let $Q$ be a cube in $\mathbb{R}^{N}$. Then, for $0<p<\infty, M_{\alpha}(\log u) \in L^{p}(Q)$ if and only if $A_{\alpha}^{2}(\log u) \in L^{p}(Q)$.

(c) Let $Q$ be a cube in $\mathbb{R}^{N}$. Then, for $1<p<\infty, \log f \in L^{p}(Q)$ if and only if $A_{\alpha}^{2}(\log u) \in L^{p}(Q)$.

(d) There exists a constant $c=c(N, \alpha)$ such that $\exp \left(c A_{\alpha}^{2}(\log u)\right) \in L_{\text {loc }}^{1}$ implies that $\mu$ and Lebesgue measure are mutually absolutely continuous.

(e) There exists a constant $C=C(N, \alpha)$ such that if $A_{\alpha}^{2}(\log u)(x)<1$ for any $x \in Q$, then $\exp \left(C M_{\alpha}^{2}(\log u)\right) \in L^{1}(Q)$.

Corollary 1.6. With the notations above, $\mu$ is singular if and only if

$$
A_{\alpha}^{2}(\log u)(x)=\infty \quad \text { a.e. }(d x) x \in \mathbb{R}^{N} .
$$

Observe that in this result the measure $\mu$ does not need to be doubling. Let us explain why. Given a positive measure $\mu$ in $\mathbb{R}^{N}$ we may consider two extensions to $\mathbb{R}_{+}^{N+1}$ : on one hand its harmonic extension that we denote by $u(x, y)$, and on the other hand the average of the measure on the cube $Q(x, y)$ centered at $x \in \mathbb{R}^{N}$ and sidelength $y>0$ that we denote by $\widetilde{u}(x, y)$. So $\widetilde{u}(x, y)=\mu(Q(x, y)) / y^{N}$. The reason why we do not need to assume any doubling hypothesis on $\mu$ in Theorem 1.5 is that Harnack's inequality holds for $u$ but not for $\widetilde{u}$ unless $\mu$ is doubling.

A proof of (a) and (b) can be given using the identity

$$
\Delta(\log u)=-\frac{|\nabla u|^{2}}{u^{2}},
$$

Green's Theorem and the traditional techniques of "sawtooth regions" (see, for instance, [35, Chapter VII]). However, (d) and (e) are harder because they really depend on the martingale subgaussian estimate of [14]. 
As it was mentioned before, Bañuelos and Moore ([6]) proved subgaussian estimates between the distribution functions of the non-tangential maximal function of a harmonic function and its area function. Their method was based on using a truncated version of a Calderón-type reproducing formula, to approximate a harmonic function by dyadic martingales, up to an error controled by its area function. To obtain the good $\lambda$-inequalities which are needed in our setting, we apply their scheme to the logarithm of a positive harmonic function. Then, a new term, $Q(\log u)$, which is analogue to the process $X_{n}$ in the martingale setting, arises but it can be estimated by the square of the area function. This is sufficient to prove (d) and (e). In our analysis it is crucial that the logarithm of a positive harmonic function shares some properties with harmonic functions, such as a submean property for its gradient. As in the martingale setting, the subgaussian estimate also leads to a Law of the Iterated Logarithm.

We need some notation. Given $y \geq 0$, the (doubly) truncated cone $\Gamma_{\alpha}(x, y)$ with vertex at $x \in \mathbb{R}^{N}$ is

$$
\Gamma_{\alpha}(x, y)=\left\{(w, t) \in \mathbb{R}_{+}^{N+1}:|w-x|<\alpha t, y<t<1\right\}
$$

and the (doubly) truncated area function of $\log u$ is defined by

$$
A_{\alpha}^{2}(\log u)(x, y)=\int_{\Gamma_{\alpha}(x, y)} t^{1-N} \frac{|\nabla u(w, t)|^{2}}{u(w, t)^{2}} d w d t
$$

Given a smooth, positive, radial function $K$ supported on $\left\{x \in \mathbb{R}^{N}:\|x\|<\rho\right\}$, with integral 1, consider

$$
Q(x, \varepsilon)=\int_{\varepsilon}^{1} \int_{\mathbb{R}^{N}} t^{1-N} K((x-w) / t) \frac{|\nabla u(w, t)|^{2}}{u(w, t)^{2}} d w d t, \quad x \in \mathbb{R}^{N} .
$$

Observe that the integral is really over the cone $\Gamma_{\rho}(x) \subset \mathbb{R}_{+}^{N+1}$. So, $Q(x, \varepsilon)$ is a smooth version of the truncated area function defined above. Actually, if $0<\beta<\rho<\alpha$, then

$$
C^{-1} A_{\beta}^{2}(\log u)(x, y)<Q(x, y)<C A_{\alpha}^{2}(\log u)(x, y),
$$

where $C$ is a numerical constant depending on $\alpha, \beta, N$ and the function $K$. Our result shows that $\log u(x, y)$ behaves very closely to $Q(x, y)$ at the set $x \in\left\{x \in \mathbb{R}^{N}: \lim _{y \rightarrow 0} u(x, y)=0\right\}$, which coincides, except for a set of Lebesgue measure 0 , with $\left\{x \in \mathbb{R}^{N}: \lim _{y \rightarrow 0} A_{\alpha}(\log u)(x, y)=\infty\right\}$ and with $\left\{x \in \mathbb{R}^{N}: \lim _{y \rightarrow 0} Q(x, y)=\infty\right\}$. 
Theorem 1.7. Let $u$ be a positive harmonic function on $\mathbb{R}_{+}^{N+1}$ and define $Q$ by (1.4). Then, there exists a constant $C=C(N, K)$ such that

$$
\limsup _{y \rightarrow 0} \frac{|\log u(x, y)+Q(x, y)|}{\sqrt{Q(x, y) \log \log Q(x, y)}} \leq C,
$$

at almost every point $x \in\left\{x \in \mathbb{R}^{N}: \lim _{y \rightarrow 0} u(x, y)=0\right\}$.

Hence,

Corollary 1.8. Let $u$ be a positive harmonic function in $\mathbb{R}_{+}^{N+1}$. Then,

$$
\lim _{y \rightarrow 0} \frac{|\log u(x, y)|}{Q(x, y)}=1
$$

at almost every point $x \in\left\{x \in \mathbb{R}^{N}: \lim _{y \rightarrow 0} u(x, y)=0\right\}$.

Also, given $\alpha>0$, there exists a constant $C=C(\alpha, N)$ such that

$$
C^{-1}<\limsup _{y \rightarrow 0} \frac{|\log u(x, y)|}{A_{\alpha}^{2}(\log u)(x, y)}<C,
$$

at almost every point $x \in\left\{x \in \mathbb{R}^{N}: \lim _{y \rightarrow 0} u(x, y)=0\right\}$.

It is worth mentioning that there is no pointwise estimate between

$$
|\log u(x, y)| \text { and } Q(x, y) \text { or } A_{\alpha}^{2}(\log u)(x, y) .
$$

Finally, we will apply these results to two different settings, the first one is related to Schwarz's Lemma and the second one to quasiconformal mappings. The problem on Schwarz's Lemma which we now describe was in fact the first motivation for our work. Let $I$ be a holomorphic mapping from the unit disc into itself. Schwarz's Lemma states that $I$ decreases hyperbolic distances, or infinitesimally that its derivative with respect to the hyperbolic metric is bounded by 1 , that is,

$$
D(I)(z)=\frac{\left(1-|z|^{2}\right)\left|I^{\prime}(z)\right|}{1-|I(z)|^{2}} \leq 1
$$

for any $z \in \mathbb{D}$. We would like to describe the best decay of the hyperbolic derivative of such non constant holomorphic functions. It is clear that a normalization of the image domain is needed. A holomorphic mapping from the unit disc onto itself is called inner if

$$
\left|\lim _{r \rightarrow 1} I\left(r e^{i \theta}\right)\right|=1, \quad \text { a.e. } e^{i \theta} \in \partial \mathbb{D} .
$$

For such mappings, W. Smith ([34], see also [1]) showed that the best radial decay of the hyperbolic derivative is governed by a quadratic condition. 
Theorem B. (a) Let $w$ be an increasing function on $[0,1], w(0)=0$. Assume

$$
\int_{0} w^{2}(t) \frac{d t}{t}<\infty
$$

Then, there exists no non-constant inner function I such that

$$
\left.\frac{\left(1-|z|^{2}\right)\left|I^{\prime}(z)\right|}{1-|I(z)|^{2}} \leq w(1-|z|)\right), \quad \text { for any } z \in \mathbb{D} \text {. }
$$

(b) Let $w$ be an increasing function on $[0,1], w(0)=0$, such that $w(t) / t^{1-\varepsilon}$ decreases for some $\varepsilon>0$. Assume

$$
\int_{0} w^{2}(t) \frac{d t}{t}=\infty
$$

Then, there exists an inner function I such that

$$
\frac{\left(1-|z|^{2}\right)\left|I^{\prime}(z)\right|}{1-|I(z)|^{2}} \leq w(1-|z|), \quad \text { for any } z \in \mathbb{D} .
$$

We will apply Theorem 1.5 to find a non-radial version of this result. As it was noticed in [1], this is really a problem on positive harmonic functions. Actually, let

$$
\tau(z)=\frac{1+z}{1-z}
$$

be a conformal mapping from the unit disc onto the right half plane. Given a holomorphic function $I: \mathbb{D} \rightarrow \mathbb{D}$, let $u$ be the real part of $\tau \circ I$. So, $u$ is a positive harmonic function and therefore the Poisson integral of a positive measure $\mu$ on $\partial \mathbb{D}$. It is clear that $I$ is inner if and only if $\mu$ is a singular measure. Also, a simple calculation shows

$$
2 \frac{\left|I^{\prime}(z)\right|}{1-|I(z)|^{2}}=\frac{|\nabla u(z)|}{u(z)}, \quad z \in \mathbb{D} .
$$

Given $\theta \in[0,2 \pi]$ and $\alpha>1$ we denote by $\Gamma_{\alpha}(\theta)$ the Stölz angle with vertex at $e^{i \theta}$, that is

$$
\Gamma_{\alpha}(\theta)=\left\{z \in \mathbb{D}:\left|z-e^{i \theta}\right| \leq \alpha(1-|z|)\right\} .
$$

We consider the following hyperbolic version of the area function

$$
\mathcal{A}_{\alpha}^{2}(I)\left(e^{i \theta}\right)=\int_{\Gamma_{\alpha}(\theta)} \frac{\left|I^{\prime}(z)\right|^{2}}{\left(1-|I(z)|^{2}\right)^{2}} d m(z),
$$

which corresponds to the usual area function once one has replaced the euclidean derivative and the Lebesgue measure by the hyperbolic derivative $D(I)(z)$ and the hyperbolic density $d m(z) /\left(1-|z|^{2}\right)^{2}$. 
Geometrically, $\mathcal{A}_{\alpha}^{2}(I)\left(e^{i \theta}\right)$ is the hyperbolic area (in the disc), counting multiplicities, of $I\left(\Gamma_{\alpha}(\theta)\right)$. With the previous notation $\mathcal{A}_{\alpha}^{2}(I)\left(e^{i \theta}\right)$ coincides with $4^{-1} A_{\alpha}^{2}(\log u)\left(e^{i \theta}\right)$. Part (a) of our next result can be understood as the hyperbolic analogous of the classical result by Calderón on the existence of radial limits at almost every point where the usual area function is finite. More concretely, since the function $I$ is bounded, Fatou's Theorem asserts that $I$ has finite radial limits at almost every point of the unit circle. However, having limits of modulus 1 means going to infinity in the hyperbolic metric of the disc. So, (a) states that the set where this occurs coincides, modulo sets of Lebesgue measure 0 , with the set where the hyperbolic area function is infinite.

Theorem 1.9. (a) Let I be a holomorphic mapping from the unit disc into itself. Fix $\alpha>0$. Then, the sets

$$
\left\{e^{i \theta} \in \partial \mathbb{D}:\left|\lim _{r \rightarrow 1} I\left(r e^{i \theta}\right)\right|<1\right\} \quad \text { and } \quad\left\{e^{i \theta} \in \partial \mathbb{D}: \mathcal{A}_{\alpha}^{2}(I)\left(e^{i \theta}\right)<\infty\right\}
$$

can only differ in a set of Lebesgue measure 0.

(b) Let $h$ be a positive function $h: \mathbb{D} \rightarrow(0, \infty)$ for which

$$
(99 / 100) h(w) \leq h(z) \leq(101 / 100) h(w),
$$

for any pair of points $z, w$ such that $|z-w| /|1-\bar{w} z| \leq 1 / 2$. Given $\alpha>0$, assume that

$$
\int_{\Gamma_{\alpha}(\theta)} h^{2}(z) d m(z)=\infty,
$$

at almost every $\theta \in[0,2 \pi)$. Assume also that, for any $0 \leq \theta<2 \pi$, the function $h\left(r e^{i \theta}\right)$ increases in $r \in[0,1)$. Then, there exists a non constant inner function I such that

$$
\frac{\left|I^{\prime}(z)\right|}{1-|I(z)|^{2}} \leq h(z), \quad \text { for any } z \in \mathbb{D} .
$$

Observe that (a) implies part (a) in Theorem B. Actually if

$$
\frac{\left(1-|z|^{2}\right)\left|I^{\prime}(z)\right|}{1-|I(z)|^{2}} \leq w(1-|z|), \quad z \in \mathbb{D},
$$

one would deduce that for any $\theta \in[0,2 \pi]$,

$$
\int_{\Gamma_{\alpha}(\theta)}\left(\frac{\left|I^{\prime}(z)\right|}{1-|I(z)|^{2}}\right)^{2} d m(z) \leq \int_{\Gamma_{\alpha}(\theta)} \frac{w^{2}(1-|z|)}{(1-|z|)^{2}} d m(z) \leq C(\alpha) \int_{0}^{1} w^{2}(t) \frac{d t}{t} .
$$

Also, part (b) of Theorem B corresponds to the case

$$
h(z)=w(1-|z|) /(1-|z|)
$$

and the crucial integral assumption on $h$ follows from the corresponding one on $w(t)$. 
As in the preceding situations, $L^{p}$ estimates between the hyperbolic area function of $I$ and the non-tangential maximal function of $\log (1-|I|)$ can be proved. Also, a Law of the Iterated Logarithm is obtained. Given a smooth, positive, radial function $K$ supported on $(-\rho, \rho)$, with integral 1, consider

$$
\mathcal{Q}\left(r e^{i \theta}\right)=\int_{\Gamma_{\rho}(\theta, r)} K((\operatorname{Arg} z-\theta) / \rho) \frac{\left|I^{\prime}(z)\right|^{2}}{\left(1-|I(z)|^{2}\right)^{2}} d m(z) \quad,
$$

where $\Gamma_{\alpha}(\theta, r)=\Gamma_{\alpha}(\theta) \cap\{|z|<r\}$ is the truncated Stölz angle with vertex at $e^{i \theta}$. This is a smooth version of the truncated hyperbolic area function given by

$$
\mathcal{A}_{\rho}(I)\left(r e^{i \theta}\right)=\int_{\Gamma_{\rho}(\theta, r)} \frac{\left|I^{\prime}(z)\right|^{2}}{\left(1-|I(z)|^{2}\right)^{2}} d m(z) .
$$

Our result is the following.

Theorem 1.10. Let I be a holomorphic mapping from the unit disc into itself and let $\mathcal{Q}$ be defined by (1.5). Then, there exists a constant $C=C(\alpha, K)$ such that

$$
\limsup _{r \rightarrow 1} \frac{\left|\log \left(1-\left|I\left(r e^{i \theta}\right)\right|^{2}\right)+\mathcal{Q}\left(r e^{i \theta}\right)\right|}{\sqrt{\mathcal{Q}\left(r e^{i \theta}\right) \log \log \mathcal{Q}\left(r e^{i \theta}\right)}} \leq C,
$$

at almost every point $e^{i \theta} \in\left\{e^{i \theta}: \lim _{r \rightarrow 1}\left|I\left(r e^{i \theta}\right)\right|=1\right\}$.

The other application of Theorem 1.5 is related to the theory of quasiconformal mappings of the upper half plane $\mathbb{R}_{2}^{+}$onto itself. Let $\rho: \mathbb{R}_{2}^{+} \rightarrow \mathbb{R}_{2}^{+}$ denote a quasiconformal mapping, $\rho(\infty)=\infty$, with complex dilatation $\sigma=\bar{\partial} \rho / \partial \rho$. Then classical results show that $\rho$ extends quasiconformally to $\mathbb{R}^{2}$, in particular its restriction to the boundary $F=\left.\rho\right|_{\mathbb{R}}$ defines a homeomorphism on $\mathbb{R}$ which is quasisymmetric, that is its derivative is a doubling measure. The converse is also true: any quasisymmetric map $F: \mathbb{R} \rightarrow \mathbb{R}$ can be extended quasiconformally to $\mathbb{R}^{2}$. There is also a one to one correspondence between such quasisymmetric mappings $F$ and conformal weldings of quasicircles, that is

$$
F(x)=\Phi[\Psi(x)] ; \quad x \in \mathbb{R}
$$

where $\Psi$ is a normalized conformal mapping of $\mathbb{R}_{2}^{-}$onto a quasidisc $\Omega$ which admits a quasiconformal extension to $\mathbb{R}_{2}^{+}$with complex dilatation $\sigma$, and $\Phi$ is a conformal mapping of $\mathbb{C} \backslash \Omega$ onto $\mathbb{R}_{2}^{+}$. A very well studied problem is to investigate the smoothness properties of the boundary correspondence $F$ in terms of the complex dilatation $\sigma$. In [13], Carleson studies this problem obtaining the conclusions of Theorem A stated at the beginning of the introduction, where $w(t)$ is, in this case, defined by

$$
w(t)=\sup _{0<\operatorname{Im} z<t}|\sigma(z)|
$$


We are interested in a result $[15$, Th. 4] proved by Dyn'kin, which we state below and that can be thought as a pointwise version of Carleson's result.

Theorem C. Let $\sigma(z)$ be the dilatation coefficient of a quasiconformal selfmapping $\Psi$ of $\mathbb{C} \backslash \mathbb{D}$, with $\Psi(\infty)=\infty$ and let

$$
g(\sigma)\left(e^{i \theta}\right)=\left(\int_{\mathbb{C} \backslash \mathbb{D}} \frac{|\sigma(\xi)|^{2}}{\left|\xi-e^{i \theta}\right|^{2}} d m(\xi)\right)^{1 / 2} .
$$

Then there exists $a>0$ such that if

$$
\exp \left(a g^{2}(\sigma)\right) \in L^{1}(\partial \mathbb{D})
$$

then $\Psi$ is absolutely continuous on $\partial \mathbb{D}$.

Dyn'kin's proof uses the representation of $\left.\Psi\right|_{\mathbb{R}}$ as the welding of a quasicircle $\Gamma$ and shows that under the hypothesis of the theorem, $\Gamma$ is rectifiable. His main tool is the pointwise estimate

$$
S\left(e^{i \theta}\right) \leq \operatorname{const} g(\sigma)\left(e^{i \theta}\right), \quad \forall e^{i \theta} \in \partial \mathbb{D}
$$

where

$$
S\left(e^{i \theta}\right)=\left(\int_{\Gamma_{\alpha}(\theta)}\left|\frac{f^{\prime \prime}(\xi)}{f^{\prime}(\xi)}\right|^{2}\right)^{1 / 2}
$$

and where $f$ represents the conformal mapping from $\mathbb{D}$ onto the inner domain bounded by $\Gamma$.

We will consider instead of $S$, the area function $A_{\alpha}^{2}(\log u)$; where $u$ is the harmonic extension of a doubling measure $\mu$, and prove an inequality similar to (1.6), which does not involve conformal techniques, and which will alow us to recover Dyn'kin's Theorem. Since we state our result on the real line, we need to impose some control on the doubling constant of $\mu$ to guarantee the convergence of its Poisson integral. More precisely we obtain:

Theorem 1.11. Let $\mu$ be a doubling measure on $\mathbb{R}$, with doubling constant $C<2 \sqrt{2}$, that is $\mu(2 I) \leq C \mu(I)$, for all intervals $I \subset \mathbb{R}$. Denote by $u=P[\mu]$ the harmonic extension of $\mu$ to $\mathbb{R}_{+}^{2}$ and let $\rho: \mathbb{R}^{2} \rightarrow \mathbb{R}^{2}$ be a quasiconformal extension of $\rho(x)=\mu[0, x]$. Then

$$
\int_{\Gamma_{\alpha}(x)} \frac{|\nabla u(z)|^{2}}{u^{2}(z)} d m(z) \leq c \int_{\mathbb{R}_{2}^{-}} \frac{|\sigma(w)|^{2}}{|w-x|^{2}} d m(w)
$$

where $\sigma$ is the complex dilatation of $\rho$ and $c=c(\alpha, C)$ is a constant depending on $\alpha$ and $C$. 
As a consequence of this theorem and Theorem 1.5, we obtain

Theorem 1.12. With the same notation as in Theorem 1.11, the following holds:

(a) There exists a constant $a>0$ such that if

$$
\exp \left(a \int_{\mathbb{R}_{2}^{-}} \frac{|\sigma(w)|^{2}}{|w-x|^{2}} d m(w)\right) \in L_{\mathrm{loc}}^{1},
$$

then $\mu$ and Lebesgue measure are mutually absolutely continuous.

(b) If $\mu$ is singular, then

$$
\int_{\mathbb{R}_{-}^{2}} \frac{|\sigma(w)|^{2}}{|w-x|^{2}} d m(w)=\infty
$$

a.e. $(d x) x \in \mathbb{R}$.

(c) Let $0<p<\infty$. If

$$
\int_{\mathbb{R}_{2}^{-}} \frac{|\sigma(w)|^{2}}{|w-x|^{2}} d m(w) \in L_{\mathrm{loc}}^{p},
$$

then $M_{\alpha}(\log u) \in L_{\mathrm{loc}}^{p}$.

The paper is organized as follows. Section 2 contains the results on dyadic doubling measures. Also an example of a positive, singular, dyadic doubling measure $\mu$, for which the square functions $X_{n}, A_{n}^{2}(\mu)$ can be given explicitely, is presented.

Section 3 is devoted to the results on positive harmonic functions. A class of positive harmonic functions, for which the square function has maximal growth, is also introduced.

Section 4 contains the proof of Theorems 1.9 and 1.10 as well as an analogous result for inner functions into hyperbolic domains in the complex plane.

In Section 5, we prove the results related to quasiconformal mapppings, in particular Theorems 1.11 and 1.12 .

Finally, in Section 6 a non-dyadic square function of a doubling measure is introduced. This section also contains some questions.

We would like to thank R. Bañuelos, J. L. Fernández and P. MacManus for several helpful conversations. 


\section{Dyadic doubling measures}

\section{The logarithmic transform of a dyadic martingale}

There is a correspondence between positive dyadic martingales and positive measures. Given a positive measure $\mu$ in $Q_{0}$, we may consider the positive dyadic martingale defined by

$$
S_{n}=\sum \frac{\mu(Q)}{|Q|} \chi_{Q}
$$

where the sum is taken over all dyadic subcubes $Q$ of $Q_{0}$ of generation $n$. Conversely, given a positive dyadic martingale $\left\{S_{n}\right\}$ we may define a positive measure $\mu$ on $Q_{0}$ by first defining it on dyadic subcubes $Q$ as

$$
\mu(Q)=S_{n}(Q)|Q|
$$

if $Q$ is of generation $n$, and extending it by standard methods. As it was mentioned at the Introduction, the martingale $S_{n}$ defined above does not seem to be well suited to study multiplicative properties of the measure $\mu$ and roughly speaking, one should consider instead its logarithm.

Let $S_{n}$ be a positive dyadic martingale on a cube $Q_{0}$ in $\mathbb{R}^{N}$. Then $\log S_{n}$ is a supermartingale. We will show that there exists a unique (except for adding constants) process $X_{n}$ such that

(a) $\log S_{n}+X_{n}$ is a dyadic martingale.

(b) $X_{n}$ is $F_{n-1}$-measurable, that is, $X_{n}$ is constant on each cube of generation $n-1$.

Let us first show the unicity. Assume that $\left\{Y_{n}\right\}$ is a dyadic martingale such that $Y_{n}$ is constant on cubes of generation $n-1, n=1,2, \ldots$. Let $Q, Q^{\prime}$ be two cubes of generation $n-1$ which are contained in the same cube $\widetilde{Q}$ of generation $n-2$. Since $Y_{n-1}(Q)$ is the arithmetic mean of $Y_{n}$ on the cubes of generation $n$ contained in $Q$, we have $Y_{n}(Q)=Y_{n-1}(Q)$. Similarly $Y_{n}\left(Q^{\prime}\right)=Y_{n-1}\left(Q^{\prime}\right)$. So, $Y_{n}(Q)=Y_{n}\left(Q^{\prime}\right)$. Hence, $Y_{n}$ is constant on cubes of generation $n-2$. Repeating the argument on cubes of generation $n-2, \ldots, 1$, one shows that $Y_{n}$ is constant.

As the following lemma asserts, the process $X_{n}$ satisfying (a) and (b) can be given explicitely. We recall the notation given in the introduction. Given a positive martingale $\left\{S_{n}\right\}$ in $Q_{0}$ and a dyadic subcube $Q$ of $Q_{0}$ of generation $n$, we denote by a.m. $(Q)$, (g.m. $(Q))$ the arithmetic (geometric) mean of the values of $S_{n}$ in the cubes of generation $(n+1)$ contained in $Q$. 
More explicitely, if $Q$ is of generation $n$, then

$$
\begin{aligned}
& \text { a.m. }(Q)=S_{n}(Q)=\frac{1}{2^{N}} \sum S_{n+1}\left(Q^{\prime}\right), \\
& \text { g.m. }(Q)=\prod S_{n+1}\left(Q^{\prime}\right)^{1 / 2^{N}}
\end{aligned}
$$

where both the sum and the product are taken over the $2^{N}$ cubes $Q^{\prime}$ of generation $(n+1)$ contained in $Q$. Consider

$$
X_{n}=\sum_{k} X_{n}\left(Q_{k}^{(n)}\right) \chi_{Q_{k}^{(n)}}, \quad n=1,2, \ldots
$$

where $\left\{Q_{k}^{(n)}: k=1, \ldots, 2^{N n}\right\}$ are the dyadic subcubes of $Q_{0}$ of generation $n$ and

$$
X_{n}\left(Q_{k}^{(n)}\right)=-\sum_{j} \log \left(\frac{\text { g.m. }\left(Q_{j}\right)}{\operatorname{a.m} .\left(Q_{j}\right)}\right),
$$

where the sum is taken over all dyadic subcubes $Q_{j}$ of $Q_{0}$ which properly contain $Q_{k}^{(n)}$. It is clear that $X_{n}$ is positive, increasing process satisfying property (b). Also, $X_{n}(Q)$ measures, roughly speaking, the error the martingale does when doubling at the dyadic cubes containing $Q$.

Lemma 2.1. Let $\left\{S_{n}\right\}$ be a positive dyadic martingale in a cube $Q_{0}$. Let $X_{n}$, $n=1,2, \ldots$, be the functions given by (2.1). Then

$$
T_{n}=\sum\left(\log S_{n}+X_{n}\right) \chi_{Q},
$$

where the sum is taken over all dyadic subcubes $\{Q\}$ of $Q_{0}$ of generation $n$, is a dyadic martingale.

Proof. Let $Q$ be a dyadic subcube of $Q_{0}$ of generation $n$ and let us denote by $\left\{Q_{k}\right\}$ the $2^{N}$ dyadic cubes of generation $n+1$ contained in $Q$. We have to show

$$
T_{n}(Q)=\frac{1}{2^{N}} \sum_{k} T_{n+1}\left(Q_{k}\right) .
$$

Since $X_{n+1}\left(Q_{k}\right)=X_{n+1}\left(Q_{j}\right)$, for any $k, j=1, \ldots, 2^{N}$ and

$$
X_{n}(Q)-X_{n+1}\left(Q_{k}\right)=\log \frac{\text { g.m. }(Q)}{\operatorname{a.m} .(Q)}
$$

the equation (2.2) is equivalent to

$$
\log S_{n}(Q)=\frac{1}{2^{N}} \sum_{k} \log S_{n+1}\left(Q_{k}\right)-\log \frac{\text { g.m. }(Q)}{\text { a.m. }(Q)} .
$$

Since a.m. $(Q)=S_{n}(Q)$, this is an identity and the lemma is proved. 
The results mentioned at the introduction relate the behaviour of $T_{n}=$ $\log S_{n}+X_{n}$ with the size of the square function of the martingale. The main observations, which will be proved below, are that when $\left\{S_{n}\right\}$ is a doubling martingale, $X_{n}$ is pointwise comparable to $\langle T\rangle_{n}^{2}$ and that $\langle T\rangle(x)$ behaves as

$$
\left(\sum_{n}\left\|\left(\log S_{n} / S_{n-1}\right) X_{Q_{n-1}(x)}\right\|_{\infty}^{2}\right)^{1 / 2}, \quad x \in Q_{0} .
$$

\section{Comparing $X_{n}$ and $\langle T\rangle_{n}^{2}$ for doubling martingales}

A positive dyadic martingale $\left\{S_{n}\right\}$ is called doubling if there exists a constant $M \geq 1$ such that

$$
M^{-1} \leq S_{n+1} / S_{n} \leq M, \quad n=1,2, \ldots
$$

The main estimate is given in the following result.

Lemma 2.2. Let $\left\{S_{n}\right\}$ be a dyadic doubling martingale in $Q_{0}$. Let $X_{n}$ be the process given by (2.1) and $T_{n}=\log S_{n}+X_{n}$ be the logarithm transform of the martingale. Then, there exists a constant $C>0$, which depends on the doubling constant of the martingale, such that

$$
\begin{gathered}
C^{-1} \leq \frac{\langle T\rangle_{n}^{2}(x)}{\sum_{k=1}^{n}\left\|\log \left(S_{k} / S_{k-1}\right) \chi_{Q_{k-1}(x)}\right\|_{\infty}^{2}} \leq C, \\
C^{-1} \leq \frac{X_{n}(x)}{\sum_{k=1}^{n}\left\|\log \left(S_{k} / S_{k-1}\right) \chi_{Q_{k-1}(x)}\right\|_{\infty}^{2}} \leq C,
\end{gathered}
$$

for any $x \in Q_{0}, n=1,2, \ldots$.

Proof. We will show that in the corresponding sums, the terms are comparable, that is,

$$
\begin{gathered}
C^{-1} \leq \frac{\left\|\left(T_{j+1}-T_{j}\right) \chi_{Q_{j}(x)}\right\|_{\infty}^{2}}{\left\|\log \left(S_{j+1} / S_{j}\right) \chi_{Q_{j}(x)}\right\|_{\infty}^{2}} \leq C, \\
C^{-1} \leq \frac{-\log \left(\text { g.m. }\left(Q_{j}\right) / \text { a.m. } .\left(Q_{j}\right)\right)}{\left\|\log \left(S_{j+1} / S_{j}\right) \chi_{Q_{j}(x)}\right\|_{\infty}^{2}} \leq C,
\end{gathered}
$$

To prove the first estimate note that $\left\|\left(T_{n}-T_{n-1}\right) \chi_{Q_{n-1}(x)}\right\|_{\infty}$ is comparable to $\max \left|T_{n}\left(Q_{j}\right)-T_{n}\left(Q_{k}\right)\right|$, where the maximum is taken over the $2^{N}$ dyadic cubes of generation $n$ contained in $Q_{n-1}(x)$. Since $X_{n}$ is predictible, this quantity is $\max \left|\log \left(S_{n}\left(Q_{j}\right) / S_{n}\left(Q_{k}\right)\right)\right|$. The estimate follows now from the fact that $S_{n}$ is a doubling martingale. 
To prove the second one we will show that given $L=2^{N}$ positive numbers $a_{1}, \ldots, a_{L}$, with $M^{-1} \leq a_{k} / a_{j} \leq M, k, j=1, \ldots, L$, there exists a constant $C=C(M, L)>0$ such that

$$
C^{-1} \leq \frac{-\log \left(\left(\prod a_{k}^{1 / L}\right) / \frac{1}{L} \sum a_{k}\right)}{\max _{j}\left(\log \left(a_{j} / \frac{1}{L} \sum a_{k}\right)\right)^{2}} \leq C .
$$

We may assume that $\sum a_{k}=L$, then we have to show that

$$
C^{-1} \leq \frac{-\log \left(\prod_{k} a_{k}^{1 / L}\right)}{\max _{k}\left(\log a_{k}\right)^{2}} \leq C .
$$

Writing $a_{k}=1+x_{k}$, this follows from the following observation.

Lemma 2.3. Let $m>-1, M>0$ and $x_{k} \in[m, M], k=1, \ldots, L$, so that

$$
\sum_{k=1}^{L} x_{k}=0
$$

Then, there exists a constant $C=C(m, M)>0$ such that

$$
C^{-1} \leq \frac{-\sum_{k=1}^{L} \log \left(1+x_{k}\right)}{\sum_{k=1}^{L} x_{k}^{2}} \leq C .
$$

Observe that $\sum_{k=1}^{L} \log \left(1+x_{k}\right) \leq 0$, because the arithmetic mean of $x_{1}, \ldots, x_{L}$ vanishes. So, Lemma 2.3 shows that the trivial estimate

$$
-\sum_{k=1}^{L} \log \left(1+x_{k}\right) \leq C \sum_{k=1}^{L}\left|x_{k}\right|,
$$

can be substantially improved when $\sum_{k=1}^{L} x_{k}=0$.

Proof of Lemma 2.3. One may assume that $x_{k}, k=1, \ldots, L$, are close to 0 , because otherwise the geometric mean of $1+x_{k}$ is bounded away from one and then there exists $C_{1}<0$ such that

$$
\sum \log \left(1+x_{k}\right) \leq C_{1}<0
$$

and the result follows in this case. So, we assume $x_{k}, k=1, \ldots, L$ are close to 0 . Using that $-\log t=1-t+O\left((1-t)^{2}\right)$ for $t$ close to 1 , one gets

$$
-\log \prod_{k=1}^{N}\left(1+x_{k}\right) \simeq 1-\prod_{k=1}^{N}\left(1+x_{k}\right) .
$$


Now, since $\sum_{k=1}^{N} x_{k}=0$, one has

$$
1-\prod_{k=1}^{N}\left(1+x_{k}\right)=-\sum_{i<j} x_{i} x_{j}+O\left(\sum_{i=1}^{N} x_{i}^{3}\right)=\frac{1}{2} \sum_{i=1}^{N} x_{i}^{2}+O\left(\sum_{i=1}^{N} x_{i}^{3}\right),
$$

because

$$
0=\left(\sum_{i=1}^{N} x_{i}\right)^{2}=\sum_{i=1}^{N} x_{i}^{2}+2 \sum_{i<j} x_{i} x_{j}
$$

This finishes the proof.

\section{Dyadic doubling measures}

Recall that a positive measure $\mu$ in $Q_{0}$ is called dyadic doubling if there exists a constant $C=C(\mu)>0$ such that $\mu(\widetilde{Q}) \leq C \mu(Q)$ for all dyadic subcubes $Q$ of $Q_{0}$, where $\widetilde{Q}$ is the smallest dyadic cube properly containing $Q$.

It is clear that dyadic doubling martingales correspond to dyadic doubling measures. In particular, given a dyadic doubling measure $\mu$ in $Q_{0}$ one may consider its corresponding martingale $\left\{S_{n}\right\}, S_{n}(Q)=\mu(Q) /|Q|$ where $Q$ is of generation $n$. Then we consider the martingale $\left\{T_{n}\right\}$ given by $T_{n}=\log S_{n}+X_{n}$, where $X_{n}$ is given in (2.1). As stated in Lemma 2.2, the square function of $T_{n}$ is comparable to

$$
\langle T\rangle^{2}(x) \cong \sum_{k=1}^{\infty} s_{k}^{2}(x)
$$

where

$$
s_{k}(x)=\max _{j=1, \ldots, 2^{N}}\left|\log \left(\frac{\mu\left(Q_{j}\right)}{\left|Q_{j}\right|} / \frac{\mu\left(Q_{k-1}(x)\right)}{\left|Q_{k-1}(x)\right|}\right)\right| .
$$

Here $Q_{k-1}(x)$ is the unique cube of generation $k-1$ which contains $x$ and $\left\{Q_{j}: j=1, \ldots, 2^{N}\right\}$ are the cubes of generation $k$ contained in $Q_{k-1}(x)$.

Now, given a dyadic doubling measure $\mu$, define $A^{2}(\mu)(x)=\sum_{k=1}^{\infty} d_{k}^{2}(x)$, where

$$
d_{k}(x)=\max _{j=1, \ldots, 2^{N}}\left\{\left|1-\frac{\mu\left(Q_{j}\right) 2^{N}}{\mu\left(Q_{k-1}(x)\right)}\right|\right\} .
$$

It is clear that $A^{2}(\mu)(x)$ is (pointwise) comparable to $\langle T\rangle^{2}(x)$. Note that in dimension $1, A^{2}(\mu)(x)$ is comparable to

$$
\sum_{k=1}^{\infty}\left(1-\frac{\mu\left(I_{k}(x)\right)}{\mu\left(I_{k}^{\prime}(x)\right)}\right)^{2}
$$

where $I_{k}^{\prime}(x)$ is the unique dyadic interval of length $2^{-k}$, such that $I_{k}(x) \cup I_{k}^{\prime}(x)$ is also dyadic. 
Given a dyadic doubling measure $\mu$ on $Q_{0}$ and a dyadic subcube $Q_{1}$ of $Q_{0}$, we consider the maximal function

$$
M\left(\log \mu, Q_{1}\right)(x)=\sup \left|\log \frac{\mu(Q)}{|Q|}-\log \frac{\mu\left(Q_{1}\right)}{\mu\left(Q_{1}\right)}\right|, \quad x \in Q_{1},
$$

where the supremum is taken over all dyadic subcubes $Q$ of $Q_{1}$ which contain $x$. Also, let us consider

$$
A^{2}\left(\mu, Q_{1}\right)(x)=\sum_{k>k_{0}} d_{k}^{2}(x), \quad x \in Q_{1},
$$

where $k_{0}$ is determined by the fact that $Q_{1}$ is a dyadic subcube of $Q_{0}$ of generation $k_{0}$. The following result will follow from the sharp good- $\lambda$ inequality proved by Chang, Wilson and Wolff, mentioned in the introduction.

Theorem 2.4. Let $\mu$ be a dyadic doubling measure in $Q_{0}$. Let $A(\mu)$ be its square function, that is,

$$
A^{2}(\mu)(x)=\sum_{k} d_{k}^{2}(x), \quad x \in Q_{0} .
$$

Then, there exist $\lambda_{0}=\lambda_{0}(\mu)$ and three universal constants $C_{1}, C_{2}, C_{3}>0$ such that for any $\lambda>\lambda_{0}$, any $k \geq C_{3}$ and any dyadic subcube $Q_{1}$ of $Q_{0}$, the following holds

(a) $\left|\left\{x \in Q_{1}: M\left(\log \mu, Q_{1}\right)(x)>k \lambda, A^{2}\left(\mu, Q_{1}\right)(x)<\lambda\right\}\right|$

$$
\leq C_{1} \exp \left(-C_{2} k^{2} \lambda\right)\left|\left\{x \in Q_{1}: M\left(\log \mu, Q_{1}\right)(x)>\lambda\right\}\right|
$$

(b) $\left|\left\{x \in Q_{1}: A^{2}\left(\mu, Q_{1}\right)(x)>k \lambda, M\left(\log \mu, Q_{1}\right)(x)<\lambda\right\}\right|$

$$
\leq C_{1} \exp \left(-C_{2} k \lambda\right)\left|\left\{x \in Q_{1}: A^{2}\left(\mu, Q_{1}\right)(x)>\lambda\right\}\right|
$$

Proof. The letters $C_{1}, C_{2}, \ldots$ will denote different constants whose value may change from line to line. Let $\left\{T_{n}\right\}$ be the dyadic martingale given in Lemma 2.1. Let $Q$ be a dyadic subcube of $Q_{0}$. We will first show the following estimate:

(2.3) $\left|\left\{x \in Q: M(\log \mu, Q)(x)>k \lambda, A^{2}(\mu)(x)<\lambda\right\}\right| \leq C_{1} \exp \left(-C_{2} \lambda k^{2}\right)|Q|$.

We may assume that $Q=Q_{0}$, then by the subgaussian estimate (1.1)

$\left|\left\{x \in Q: \sup _{n}\left|\log S_{n}(x)+X_{n}(x)\right|>k \lambda,\langle T\rangle(x)<\lambda\right\}\right| \leq C_{1} \exp \left(-C_{2} k^{2}\right)|Q|$,

for any $k>1$. Recall that $X_{n}$ is pointwise comparable to $\langle T\rangle_{n}^{2}$. So, if $k$ is sufficiently large, one deduces

$$
\left|\left\{x \in Q: \sup _{n}\left|\log S_{n}(x)\right|>k \lambda,\langle T\rangle(x)<\lambda^{1 / 2}\right\}\right| \leq C_{1} \exp \left(-C_{2} \lambda k^{2}\right)|Q| .
$$

Now, since $\langle T\rangle(x)$ is comparable to $A(\mu)(x),(2.3)$ holds. 
Next we prove (a). Given the cube $Q_{1}$, let $\left\{Q_{j}: j=2, \ldots\right\}$ be the maximal dyadic subcubes of $Q_{1}$ which satisfy

$$
\left|\log \frac{\mu\left(Q_{j}\right)}{\left|Q_{j}\right|}-\log \frac{\mu\left(Q_{1}\right)}{\left|Q_{1}\right|}\right|>\lambda .
$$

Since $\mu$ is doubling, the maximality implies

$$
\left|\log \frac{\mu\left(Q_{j}\right)}{\left|Q_{j}\right|}-\log \frac{\mu\left(Q_{1}\right)}{\left|Q_{1}\right|}\right| \leq \lambda+C,
$$

where $C=C(\mu, N)$ is a constant depending on the doubling constant of $\mu$ and on the dimension. Observe that

$$
\left\{x \in Q_{1}: M\left(\log \mu, Q_{1}\right)(x)>\lambda\right\}=\bigcup_{j} Q_{j} .
$$

Now, if $\lambda$ and $k$ are sufficiently large, we have

$$
\begin{aligned}
&\left\{x \in Q_{1}: M\left(\log \mu, Q_{1}\right)(x)>k \lambda, \quad A^{2}\left(\mu, Q_{1}\right)(x)<\lambda\right\} \subseteq \\
& \subseteq \bigcup_{j}\left\{x \in Q_{j}: M\left(\log \mu, Q_{j}\right)(x)>(k-2) \lambda, \quad A^{2}\left(\mu, Q_{j}\right)(x)<\lambda\right\} .
\end{aligned}
$$

So, applying estimate (2.3) in each $Q_{j}$, one has

$$
\begin{aligned}
& \left|\left\{x \in Q_{1}: M\left(\log \mu, Q_{1}\right)(x)>k \lambda, \quad A^{2}\left(\mu, Q_{1}\right)(x)<\lambda\right\}\right| \\
& \quad \leq C_{3} e^{-C_{4} k^{2} \lambda} \sum_{j}\left|Q_{j}\right|=C_{3} e^{-C_{4} k^{2} \lambda}\left|\left\{x \in Q_{1}: M\left(\log \mu, Q_{1}\right)(x)>\lambda\right\}\right|,
\end{aligned}
$$

which is (a).

Given two dyadic cubes $Q_{2} \subset Q_{1}$, consider

$$
A^{2}\left(\mu, Q_{2}, Q_{1}\right)=\sum \max _{j}\left(1-\frac{2^{N} \mu\left(Q_{j}\right)}{\mu(Q)}\right)^{2},
$$

where the sum is taken over all dyadic cubes $Q$ such that $Q_{2} \subset Q \subset Q_{1}$ and the maximum is over the dyadic cubes $Q_{j}$ of $Q$ with $l\left(Q_{j}\right)=l(Q) / 2$. Fix the cube $Q_{1}$ and consider the maximal dyadic subcubes of $Q_{1}$ which satisfy

$$
A^{2}\left(\mu, Q_{j}, Q_{1}\right)>\lambda \text {. }
$$

It is clear that the maximality implies

$$
A^{2}\left(\mu, Q_{j}, Q_{1}\right) \leq \lambda+C,
$$

where $C$ is a constant depending on the doubling constant of $\mu$ and on the dimension. Also

$$
\left\{x \in Q_{1}: A^{2}\left(\mu, Q_{1}\right)(x)>\lambda\right\}=\bigcup Q_{j}
$$


Next for each $Q_{j}$ we consider the maximal dyadic subcubes $\left\{Q_{j, l}\right\}_{l}$ such that

$$
A^{2}\left(\mu, Q_{j, l}, Q_{1}\right)>k \lambda .
$$

and consider the subfamily $G_{1}$ of $\left\{Q_{j, l}\right\}_{j, l}$ composed by those cubes satisfying

$$
\left|\log \frac{\mu\left(Q_{j, l}\right)}{\left|Q_{j, l}\right|}-\log \frac{\mu\left(Q_{1}\right)}{\left|Q_{1}\right|}\right|<\lambda .
$$

Observe now that

$$
\left\{x \in Q_{1}: A^{2}\left(\mu, Q_{1}\right)(x)>k \lambda, M\left(\log \mu, Q_{1}\right)(x)<\lambda\right\} \subset \bigcup Q_{j, l}
$$

where the union is taken over all cubes in the family $G_{1}$, and that on these cubes $Q_{j, l} \in G_{1}$ we have

$$
\left|\log S_{n}+X_{n}\right|>C_{1}(k-1) \lambda
$$

and

$$
\langle T\rangle \leq C_{2}(k \lambda+C)^{1 / 2}
$$

since $\langle T\rangle_{n}^{2}$ and $X_{n}$ are pointwise comparable to $A_{n}^{2}$. Hence, the estimate (1.1) of Chang, Wilson and Wolff applied to the cubes $Q_{j, l} \in G_{1}$ give

$$
\sum_{l}\left|Q_{j, l}\right| \leq C_{1} e^{-C_{2} k \lambda}\left|Q_{j}\right|
$$

So,

$$
\begin{aligned}
\mid\{x & \left.\in Q_{1}: A^{2}\left(\mu, Q_{1}\right)(x)>k \lambda, \quad M\left(\log \mu, Q_{1}\right)(x)<\lambda\right\} \mid \\
& \leq C_{1} e^{-C_{2} k \lambda} \sum\left|Q_{j}\right|=C_{1} e^{-C_{2} k \lambda}\left|\left\{x \in Q_{1}: A^{2}(\mu)\left(x, Q_{1}\right)>\lambda\right\}\right|
\end{aligned}
$$

Next result is deduced from the good- $\lambda$ inequalities given in Theorem 2.4.

Theorem 2.5. Let $\mu$ be a dyadic doubling measure in $Q_{0}$. Let $\mu=f d x+\mu_{s}$, where $f d x, \mu_{s}$ are its absolutely continuous and singular part with respect to Lebesgue measure. Then,

(a) The sets $\left\{x \in Q_{0}: f(x)>0\right\}$ and $\left\{x \in Q_{0}: A^{2}(\mu)(x)<\infty\right\}$ can only differ in a set of Lebesgue measure 0.

(b) $\mu$ is singular if and only if $A^{2}(\mu)(x)=\infty$, a.e. $(d x) x \in Q_{0}$.

(c) For $0<p<\infty, M(\log \mu) \in L^{p}\left(Q_{0}\right)$ if and only if $A^{2}(\mu) \in L^{p}\left(Q_{0}\right)$.

(d) For $1<p<\infty, \log f \in L^{p}\left(Q_{0}\right)$ if and only if $A^{2}(\mu) \in L^{p}\left(Q_{0}\right)$.

(e) There exists a constant $C=C(N)>0$ such that $\exp \left(C A^{2}(\mu)\right) \in L^{1}\left(Q_{0}\right)$ implies that $\mu$ and Lebesgue measure are mutually absolutely continuous.

(f) There exists a constant $c=c(N)>0$ such that if $A(\mu)(x) \leq 1$ for any $x \in Q_{0}$, then $\exp \left(c M^{2}(\log \mu)\right) \in L^{1}\left(Q_{0}\right)$. 
The two following elementary results, whose proof is omitted, will be used in the proof of Theorem 2.5.

Lemma 2.6. Let $f, g$ be two measurable functions in $Q_{0}$. Assume that there exist constants $C<1$ and $K>1$ such that for any dyadic subcube $Q$ of $Q_{0}$ and for any $\lambda>\lambda_{0}(C, K, Q)$, one has

$$
|\{x \in Q: f(x)>K \lambda, g(x)<\lambda\}| \leq C|\{x \in Q: f(x)>\lambda\}| .
$$

Then $f(x)<\infty$ at almost every point $x \in\left\{x \in Q_{0}: g(x)<\infty\right\}$

Lemma 2.7. Let $f, g$ be two measurable positive functions on $Q_{0}$. Assume that there exist constants $C<1$ and $K>1$ such that for any $\lambda>\lambda_{0}(C, K)$, one has

$$
\left|\left\{x \in Q_{0}: f(x)>K \lambda, g(x)<\lambda\right\}\right| \leq C\left|\left\{x \in Q_{0}: f(x)>\lambda\right\}\right| .
$$

Let $0<p<\infty$. Then there exists a constant $M=M(C, K)>0$ such that

$$
\|f\|_{p}^{p}<M\left(\|g\|_{p}^{p}+1\right) .
$$

Proof of Theorem 2.5. Note that

$$
\left\{x \in Q_{0}: f(x)>0\right\} \stackrel{\text { a.e. }}{=}\left\{x \in Q_{0}: \lim _{|Q| \rightarrow 0} \frac{\mu(Q)}{|Q|}>0\right\} \text {. }
$$

where the limit is taken over dyadic cubes $Q$ which contain $x$. Also the symbol $\stackrel{\text { a.e. }}{=}$ means that the two sets differ on a set of Lebesgue measure 0 . Now, we apply Lemma 2.6 to the functions

$$
\begin{aligned}
& f(x)=M\left(\log \mu, Q_{0}\right)(x) \\
& g(x)=A^{2}(\mu)(x) .
\end{aligned}
$$

The hypothesis in Lemma 2.6 follows from (a) and (b) in Theorem 2.4. This finishes the proof of (a). Part (b) follows from (a). Part (c) follows from the estimates in Theorem 2.4 and Lemma 2.7. Part (d) follows from (c). Actually, it is clear that $\sup \left\{\log ^{+}(\mu(Q) /|Q|): x \in Q\right\}$ is in $L^{p}\left(Q_{0}\right)$. Since $\mu(Q) /|Q| \geq(1 /|Q|) \int_{Q} f$, Jensen's inequality gives

$$
-\log \left(\frac{\mu(Q)}{|Q|}\right) \leq-\frac{1}{|Q|} \int_{Q} \log f .
$$

Hence, $\sup \left\{\log ^{-}(\mu(Q) /|Q|): x \in Q\right\}$ is bounded by the Hardy-Littlewood maximal function of $|\log f|$. This gives one implication. To prove the converse, observe that $\log ^{+} f$ is in $L^{p}\left(Q_{0}\right)$ and at almost every point $x$ where $f(x)<1$, one has $-\log f(x) \leq \sup \{|\log (\mu(Q) /|Q|)|: x \in Q\}$. 
We now prove (e). It is sufficient to show that

$$
N(x)=\sup \left\{\frac{\mu(Q)}{|Q|},\left(\frac{\mu(Q)}{|Q|}\right)^{-1}\right\} \in L^{1}\left(Q_{0}\right)
$$

where the supremum is taken over all dyadic cubes which contain $x$. Let

$$
M(x)=\sup \left|\log \frac{\mu(Q)}{|Q|}\right| \text {. }
$$

Since $e^{M}=N$, we will show that $e^{M} \in L^{1}\left(Q_{0}\right)$. Observe that

$$
\begin{aligned}
& \int_{Q_{0}}\left(e^{M}-1\right) d x= \\
& \quad=\int_{0}^{\infty} e^{\lambda}|\{M>\lambda\}| d \lambda=3 \int_{0}^{\infty} e^{3 \lambda}|\{M>3 \lambda\}| d \lambda \leq \\
& \quad \leq 3 \int_{0}^{\infty} e^{3 \lambda}\left|\left\{M>3 \lambda, A<\varepsilon_{1} \lambda^{1 / 2}\right\}\right| d \lambda+3 \int_{0}^{\infty} e^{3 \lambda}\left|\left\{A>\varepsilon_{1} \lambda^{1 / 2}\right\}\right| d \lambda
\end{aligned}
$$

where $0<\varepsilon_{1}<1$ is a constant to be fixed later. Using the estimate (a) in Theorem 2.4 we bound the first term by

$$
3 C_{1} \int_{0}^{\infty} \exp \left(\left(3-C_{2} / \varepsilon_{1}^{2}\right) \lambda\right)|\{M>\lambda\}| d \lambda \leq 3 C_{1} \int_{Q_{0}} M
$$

if $\varepsilon_{1}$ is chosen small enough to guarantee $3-C_{2} / \varepsilon_{1}^{2}<0$. The second term is

$$
3 \int_{0}^{\infty} e^{3 \lambda}\left|\left\{A^{2}>\varepsilon_{1}^{2} \lambda\right\}\right| d \lambda=\int_{Q_{0}} \exp \left(3 A^{2} / \varepsilon_{1}^{2}\right) d x .
$$

So, the result follows taking $C=3 / \varepsilon^{2}$. The proof of (f) is similar and we omit it.

It should be observed that part (e) of Theorem 2.5 implies part (a) of Theorem A mentioned in the introduction. Recall that $w(t)$ was defined as

$$
w(t)=\sup \max \left|\frac{\mu(\widetilde{Q}) 2^{N}}{\mu(Q)}-1\right|,
$$

where the supremum is taken over all dyadic cubes $Q$ of sidelength smaller than $t$ and the maximum is over the $2^{N}$ dyadic subcubes $\widetilde{Q}$ contained in $Q$ of sidelength $l(Q) / 2$. Then

$$
A^{2}(\mu)(x) \leq \sum_{n=1}^{\infty} w^{2}\left(2^{-n}\right) \leq \int_{0}^{1} w^{2}(t) \frac{d t}{t} .
$$

So, if the last integral converges, the square function $A^{2}(\mu)$ is bounded and it follows from (e) that $\mu$ must be absolutely continuous. 
The hypothesis in part (e) of Theorem 2.5 is closely related to the space BMO. Let $Q_{0}$ be a cube in $\mathbb{R}^{N}$. The space dyadic $\operatorname{BMO}\left(Q_{0}\right)$ consists on the integrable functions $H$ defined on $Q_{0}$ for which

$$
\|H\|_{\mathrm{BMO}}=\sup \frac{1}{|Q|} \int_{Q}\left|H-H_{Q}\right|<\infty,
$$

where

$$
H_{Q}=\frac{1}{|Q|} \int_{Q} H
$$

and the supremum is taken over all dyadic subcubes $Q$ of $Q_{0}$. Chebyshev's inequality gives that

$$
\left|\left\{x \in Q:\left|H(x)-H_{Q}\right|>\lambda\right\}\right| \leq \frac{1}{\lambda}|Q| .
$$

However, the fact that the BMO condition is given at all scales, implies a stronger estimate. This is the John-Nirenberg Theorem that states that there exist constants $C_{1}, C_{2}>0$ such that for any dyadic cube $Q$ and any $H$ in dyadic BMO, one has

$$
\left|\left\{x \in Q:\left|H(x)-H_{Q}\right|>\lambda\right\}\right| \leq C_{1} \exp \left(-C_{2} \lambda /\|H\|_{\mathrm{BMO}}\right)|Q| .
$$

Given a measurable function $H$ defined on $Q_{0}$, let $\varepsilon(H)$ be the infimum of $\varepsilon>0$ for which

$$
\sup \frac{1}{|Q|} \int_{Q} \exp \left(\varepsilon^{-1}\left|H(x)-H_{Q}\right|\right)<\infty,
$$

where the supremum is taken over all dyadic subcubes $Q$ of $Q_{0}$. The JohnNirenberg Theorem implies that $H \in \mathrm{BMO}$ if and only if $\varepsilon(H)<\infty$. Garnett and Jones $([20,21])$ proved that there exists a constant $C=C(N)>0$ such that

$$
\begin{aligned}
C^{-1} \varepsilon(H)< & \operatorname{dist}_{\mathrm{BMO}}\left(H, L^{\infty}\right)= \\
& =\inf \left\{\|H-G\|_{B M O}: G \in L^{\infty}\right\}<C \varepsilon(H) .
\end{aligned}
$$

Corollary 2.8. There exists a constant $\varepsilon_{0}=\varepsilon_{0}(N)$ such that if $\mu$ is a dyadic doubling measure on $Q_{0}$ and $\varepsilon\left(A^{2}(\mu)(x)\right) \leq \varepsilon_{0}$, then $\mu$ is absolutely continuous. Moreover $\mu=f d x$ with $f, 1 / f \in L^{1}\left(Q_{0}\right)$.

Proof. Applying (2.4), we have

$$
A^{2}(\mu)=H_{1}+H_{2},
$$

where $H_{1} \in L^{\infty}$ and $\left\|H_{2}\right\|_{\text {BMO }}$ is small if $\varepsilon_{0}$ is chosen sufficiently small. Thus, the John-Nirenberg Theorem tells $e^{C H_{2}} \in L^{1}\left(Q_{0}\right)$ if $C<C\left(\varepsilon_{0}\right) \rightarrow 0$ as $\varepsilon \rightarrow 0$. Then the corollary follows from part (e) of Theorem 2.5. 
The quadratic condition given in (b) of Theorem 2.5 characterizing singular measures is also sharp in the following sense.

Proposition 2.9. Let $h: \mathbb{R}_{+}^{2} \rightarrow(0, \infty)$ be a positive function such that if $B \subset \mathbb{R}_{+}^{2}$ is a disc with $2 B \subset \mathbb{R}_{+}^{2}$, then

$$
\sup _{B} h \leq \frac{101}{100} \inf _{B} h .
$$

Fixed $\alpha>0$, assume

$$
\int_{\Gamma_{\alpha}(x)} h^{2}(z) \frac{d m(z)}{(\operatorname{Imz})^{2}}=\infty,
$$

at almost every $x \in \mathbb{R}$. Then, there exists a positive, doubling, singular measure $\mu$ such that

$$
\left|\frac{\mu(I)}{\mu\left(I^{\prime}\right)}-1\right| \leq C h\left(x_{I}+i|I|\right),
$$

for any interval $I \subset \mathbb{R},|I| \leq 1$. Here $I^{\prime}$ is an interval adjacent to $I$ of the same sidelength and $x_{I}$ is the center of $I$.

Proof. The construction of the measure follows the ideas in [13] and [24]. We will define $\mu$ by giving its mass on intervals of the form

$$
J_{k}^{(n)}=\left[k 4^{-n},(k+1) 4^{-n}\right),
$$

where $k \in \mathbb{Z}$ and $n=0,1,2, \ldots$ The intervals $\left\{J_{k}^{(n)}: k \in \mathbb{Z}\right\}$ are called of generation $n$. We may assume that $h \leq 1 / 8$. Set $\mu\left(J_{k}^{(0)}\right)=1, k \in \mathbb{Z}$, and, by induction, assume $\mu\left(J_{k}^{(n)}\right)$ has been defined satisfying

$$
\left(1+h\left(J_{k}^{(n)}\right)\right)^{-1} \leq \frac{\mu\left(J_{k}^{(n)}\right)}{\mu\left(J_{k+1}^{(n)}\right)} \leq 1+h\left(J_{k}^{(n)}\right), \quad k \in \mathbb{Z},
$$

where $h\left(J_{k}^{(n)}\right)=h\left(k 4^{-n}+i 4^{-n}\right)$. We now proceed to define $\mu\left(J_{k}^{(n+1)}\right), k \in \mathbb{Z}$.

Let $J, J^{\prime}$ be two consecutive intervals of generation $n$. We divide $J$ into four intervals $J_{1}, \ldots, J_{4}$ of generation $n+1$ and similarly $J^{\prime}$ into $J_{1}^{\prime}, \ldots, J_{4}^{\prime}$. Let $J^{\prime}$ be to the right of $J$ and let the numbering be from the left to the right. Let $\widetilde{h}(J)=\min \left\{h\left(J_{3}\right), h\left(J_{4}\right), h\left(J_{1}^{\prime}\right), h\left(J_{2}^{\prime}\right)\right\}$ and $\alpha(J)=(4+\widetilde{h}(J))^{-1}$ so that

$$
\frac{1+\alpha(J) \widetilde{h}(J)}{1-\alpha(J) \widetilde{h}(J)}=1+\widetilde{h}(J) / 2
$$


Assume $\mu(J) \leq \mu\left(J^{\prime}\right)$. Then, set

$$
\begin{aligned}
\mu\left(J_{3}\right) & =(1-\alpha(J) \widetilde{h}(J)) \frac{\mu(J)}{4}, \\
\mu\left(J_{4}\right) & =(1+\alpha(J) \widetilde{h}(J)) \frac{\mu(J)}{4}, \\
\mu\left(J_{1}^{\prime}\right) & =(1-\alpha(J) \widetilde{h}(J)) \frac{\mu\left(J^{\prime}\right)}{4}, \\
\mu\left(J_{2}^{\prime}\right) & =(1+\alpha(J) \widetilde{h}(J)) \frac{\mu\left(J^{\prime}\right)}{4} .
\end{aligned}
$$

If $\mu(J)>\mu\left(J^{\prime}\right)$, we change all signes in the above formula. This formula defines the mass of $\mu$ over intervals of generation $n+1$. Next we show that

$$
(1+h(I))^{-1} \leq \frac{\mu(I)}{\mu\left(I^{\prime}\right)} \leq 1+h(I),
$$

for any pair of consecutive intervals $I, I^{\prime}$ of generation $n+1$. The identity (2.7) gives (2.8) when $I, I^{\prime}$ are $J_{3}, J_{4}$ or $J_{1}^{\prime}, J_{2}^{\prime}$. If $I=J_{4}$ and $I^{\prime}=J_{1}^{\prime}$, we have to show

$$
(1+\widetilde{h}(J))^{-1} \leq \frac{(1+\alpha(J) \widetilde{h}(J)) \mu(J)}{(1-\alpha(J) \widetilde{h}(J)) \mu\left(J^{\prime}\right)} \leq 1+\widetilde{h}(J) .
$$

Since $\mu(J) \leq \mu\left(J^{\prime}\right)$, the right-hand inequality follows from (2.7). To prove the left-hand inequality, observe that, by induction, $\mu\left(J^{\prime}\right) \leq(1+h(J)) \mu(J)$. Thus, it is sufficient to prove

$$
\frac{1+h(J)}{1+\widetilde{h}(J)} \leq 1+\frac{1}{2} \widetilde{h}(J),
$$

which follows from the regularity assumption on the function $h$. Finally, we need to show that

$$
\frac{1+\alpha(J) \widetilde{h}(J)}{1-\alpha\left(J^{\prime}\right) \widetilde{h}\left(J^{\prime}\right)} \leq 1+\min \left\{\widetilde{h}(J), \widetilde{h}\left(J^{\prime}\right)\right\} .
$$

Since $\alpha(J), \alpha\left(J^{\prime}\right)$ are close to $1 / 4$, this follows from the regularity assumption on $h$.

So by induction we have defined the mass of $\mu$ over all intervals of the form (2.6) and it satisfies (2.8) for such intervals. Next, we will show that it also holds for any interval, that is,

$$
1+C^{-1} h(x-t+i t) \leq \frac{\mu(x-t, x)}{\mu(x, x+t)} \leq 1+C h(x-t+i t) .
$$

Assume $x=0$ and let $4^{-n-1} \leq t<4^{-n}$. Let $(0, t)=\cup I_{k}$, where $\left\{I_{k}\right\}$ are pairwise disjoint intervals of the form (2.6) with decreasing length. Hence $\left|I_{1}\right|=4^{-n-1}$. 
Now

$$
\mu\left(I_{k}\right)=\mu\left(I_{1}\right) 4^{n+1-m(k)}\left(1+O\left(h\left(I_{1}\right)\right)\right)^{m(k)-n-1},
$$

where $I_{k}$ is of generation $m(k)$. Since $t=\sum 4^{-m(k)}$, we have

$$
\mu(0, t)-t \mu\left(I_{1}\right) 4^{n+1}=\mu\left(I_{1}\right) 4^{n+1} t O\left(h\left(I_{1}\right)\right) .
$$

The same argument applies to $\mu(-t, 0)$ and (2.9) follows.

To show that $\mu$ is singular, let $x \in \mathbb{R}$ and $I_{n}(x)$ be the unique interval of generation $n$ containing $x$. The assumption on $h$ gives that

$$
\sum_{n} h^{2}\left(I_{n}(x)\right)=\infty \text {, a.e. } x \in \mathbb{R} \text {. }
$$

Observe that

$$
\mu\left(I_{n}(x)\right)=4^{-n} \prod_{k=1}^{n}\left(1 \pm \alpha_{k}\left(I_{k}(x)\right) h\left(I_{k}(x)\right)\right) .
$$

The signes + and - occur in equal number in the construction. Since $\alpha_{k}\left(I_{k}(x)\right)$ is close to $1 / 4$, the law of large numbers and (2.10) give that at almost every point $x \in \mathbb{R}$, one has

$$
\lim _{n \rightarrow \infty} \frac{\mu\left(I_{n}(x)\right)}{4^{-n}}=0 .
$$

Hence the measure $\mu$ is singular. This finishes the proof.

\section{Law of the iterated logarithm for dyadic doubling measures}

The subgaussian estimate (1.1) of Chang, Wilson and Wolff implies the law of the iterated logarithm for dyadic martingales, that is, if $\left\{T_{n}\right\}$ is a dyadic martingale and $\langle T\rangle_{n}$ is its (truncated) square function, one has

$$
\limsup _{n \rightarrow \infty} \frac{\left|T_{n}(x)\right|}{\langle T\rangle_{n}(x) \sqrt{2 \log \log \langle T\rangle_{n}(x)}} \leq 1
$$

a.e. $x \in\left\{x \in Q_{0}: \sup _{n}\left|T_{n}(x)\right|=\infty\right\}$.

We will apply it to the dyadic martingale $T_{n}=\log S_{n}+X_{n}$, given in Lemma 2.1. When the measure is doubling, $\left\{T_{n}\right\}$ has bounded increments and it is known that, in this situation, we also have

$$
\limsup _{n \rightarrow \infty} \frac{\left|T_{n}(x)\right|}{\langle T\rangle_{n}(x) \sqrt{2 \log \log \langle T\rangle_{n}(x)}} \geq C>0,
$$

a.e. $x \in\left\{x \in Q_{0}: \sup _{n}\left|T_{n}(x)\right|=\infty\right\}$. See [4]. Using Lemma 2.2 to estimate $\langle T\rangle_{n}(x)$, we deduce Theorem 1.3 and therefore Corollary 1.4. Also, the rate of convergence in the last two results can not be improved. To show this, let us introduce in dimension 1 a well known dyadic doubling measure. 


\section{An example}

Let $I_{0}=[0,1]$ be the unit interval in the real line. We will define a measure $\mu$ by specifying its mass on dyadic intervals. Let $0 \leq \lambda<1$ and let $\mu\left(I_{0}\right)=1$. Assume $\mu(I)$ has been defined on the dyadic intervals of generation $k$.

We now proceed to define it on the intervals of the next generation. Given a dyadic subinterval $I$ of $I_{0}$ of generation $k$, let $I_{+}\left(I_{-}\right)$be its right (left) half, that is, $I_{+} I_{-}$are the two intervals of generation $k+1$ contained in $I$. Then set

$$
\begin{aligned}
& \mu\left(I_{+}\right)=\frac{1+\lambda}{2} \mu(I), \\
& \mu\left(I_{-}\right)=\frac{1-\lambda}{2} \mu(I) .
\end{aligned}
$$

It is clear that if $I$ is a dyadic interval of generation $n$,

$$
\frac{\mu(I)}{|I|}=(1+\lambda)^{k}(1-\lambda)^{n-k}
$$

where $k$ is the number of dyadic intervals containing $I$ which are at righthand position.

Actually, this is a well known example. Let us consider a biassed coin, that is, a sequence of equally distributed and independent random variables $\left\{X_{n}\right\}$ such that

$$
p\left\{X_{n}=0\right\}=\frac{(1-\lambda)}{2} \text { and } p\left\{X_{n}=1\right\}=\frac{(1+\lambda)}{2} .
$$

Now, consider the random variable

$$
X=\sum_{n=1}^{\infty} 2^{-n} X_{n}
$$

If $I$ is a dyadic interval of generation $n$, we have

$$
\mu(I)=p\{X \in I\} .
$$

Equivalently, if the left end of $I$ is

$$
a=\sum_{k=1}^{\infty} \frac{t_{k}}{2^{k}}
$$

where $t_{k}=0$ or 1 , then $\mu(I)=p\left\{X_{1}=t_{1}, \ldots, X_{n}=t_{n}\right\}$. 
By construction,

$$
\frac{\mu\left(I_{+}\right)}{\mu\left(I_{-}\right)}=\frac{1+\lambda}{1-\lambda}
$$

for any pair of dyadic intervals $I_{+}, I_{-}$of the same generation contained in a interval of the previous one. Hence $\mu$ is a dyadic doubling measure. Also, when $0<\lambda<1, \mu$ is singular. Actually, the measure $\mu$ is concentrated in a set of Haussdorff dimension

$$
\gamma=\frac{(1+\lambda) \log \frac{2}{1+\lambda}+(1-\lambda) \log \frac{2}{1-\lambda}}{2 \log 2}
$$

that satisfies $\gamma<1$ if $0<\lambda<1$. See [22] for a more general result.

Let $I$ be a dyadic interval of length $2^{-n}$. Then, an easy calculation shows

$$
\begin{aligned}
X_{n}(I) & =-\frac{n}{2} \log \left(1-\lambda^{2}\right), \\
A_{n}^{2}(\mu)(I) & =n \lambda^{2} .
\end{aligned}
$$

So, Theorem 1.3 and Corollary 1.4 state that at almost $(d x)$ every point $x \in[0,1]$, one has

$$
\begin{gathered}
C^{-1}(\lambda) \leq \limsup _{n \rightarrow \infty} \frac{\left|\log \left(\frac{\mu\left(I_{n}(x)\right)}{\left|I_{n}(x)\right|}\right)-\frac{n}{2} \log \left(1-\lambda^{2}\right)\right|}{\sqrt{n \log \log n}} \leq C(\lambda), \\
\lim _{n \rightarrow \infty} \frac{\log \frac{\mu\left(I_{n}(x)\right)}{\left|I_{n}(x)\right|}}{\frac{n}{2} \log \left(1-\lambda^{2}\right)}=1
\end{gathered}
$$

Next, we show that these estimates are best possible. By the law of large numbers

$$
\lim _{n \rightarrow \infty} \frac{A_{n}(x)}{n}=1 / 2, \quad \text { a.e. }(d x) x \in[0,1]
$$

and

$$
\limsup _{n \rightarrow \infty} \frac{\left|A_{n}(x)-n / 2\right|}{\sqrt{n \log \log n}}=1, \quad \text { a.e. }(d x) x \in[0,1]
$$

Now,

$$
\log \frac{\mu\left(I_{n}(x)\right)}{\left|I_{n}(x)\right|}=A_{n}(x) \log (1+\lambda)+\left(n-A_{n}(x)\right) \log (1-\lambda) .
$$

So, (2.11) and (2.12) are equivalent to (2.13) and (2.14), except for the value of the constant $C(\lambda)$. 


\section{Positive harmonic functions}

\section{Logarithms of positive harmonic functions}

Let $u$ be the Poisson extension of a positive measure $\mu$. As it was explained in the Introduction, the area function associated to the harmonic function $u$ is not well suited to treat the doubling properties of the measure, and one should consider instead the subharmonic function $-\log u$.

The natural area function associated to a subharmonic function $v$ in $\mathbb{R}^{N}$ is

$$
A_{\alpha}^{2}(v)\left(x_{0}\right)=\int_{\Gamma_{\alpha}\left(x_{0}\right)} \Delta v(x, y) y^{1-N} d x d y .
$$

Observe that if $v=u^{2}$, where $u$ is harmonic, one obtains the usual area function of $u$. Uchiyama ([38]), extending previous work of McConnell ([30]), showed that for any $1<p<\infty$,

$$
\left\|A_{\alpha}(v)\right\|_{p} \leq C(p, N, \alpha, \beta)\left\|N_{\beta}(v)\right\|_{p} .
$$

Let $u$ be a positive harmonic function in $\mathbb{R}_{+}^{N+1}$. In our case a simple calculation shows

$$
|\nabla(\log u)|^{2}=-\Delta(\log u)=\frac{|\nabla u|^{2}}{u^{2}} .
$$

So, the natural square function for $\log u$ is

$$
A_{\alpha}^{2}(\log u)\left(x_{0}\right)=\int_{\Gamma_{\alpha}\left(x_{0}\right)} \frac{|\nabla u(x, y)|^{2}}{u(x, y)^{2}} y^{1-N} d x d y .
$$

We consider the non-tangential maximal function of $\log u$,

$$
M_{\alpha}(\log u)\left(x_{0}\right)=\sup \left\{|\log u(x, y)|:(x, y) \in \Gamma_{\alpha}\left(x_{0}\right)\right\} .
$$

We now define the local versions of the functions above. Given a cube $Q \subset \mathbb{R}^{N}$ centered at $x_{Q}$ and $x_{0} \in Q$, define

$$
\begin{aligned}
M_{\alpha}(\log u, Q)\left(x_{0}\right) & =\sup \left\{\left|\log u(x, y)-\log u\left(x_{Q}, l(Q)\right)\right|:\right. \\
\left.(x, y) \in \Gamma_{\alpha}\left(x_{0}\right), \quad 0<y<l(Q)\right\}, & \\
A_{\alpha}^{2}(\log u, Q)\left(x_{0}\right)= & \int_{\Gamma_{\alpha}\left(x_{0}\right) \cap Q} \frac{|\nabla u(x, y)|^{2}}{u(x, y)^{2}} y^{1-N} d x d y .
\end{aligned}
$$

Now, the good $\lambda$-inequalities relating these two functions are described in the following result, whose proof will be given after the next section. 
Theorem 3.1. Let $u$ be a positive harmonic function in the upper half space $\mathbb{R}_{+}^{N+1}$. Then there exists a constant $K_{0}>1$ and positive constants $C_{1}, C_{2}$ such that for any cube $Q \subset \mathbb{R}^{N}$, for all $K>K_{0}$ and $\lambda>1$, one has

(a) $\left|\left\{x \in Q: M_{\alpha}(\log u, Q)(x)>K \lambda, A_{\alpha}^{2}(\log u, Q)(x)<\lambda\right\}\right|$

$$
\leq C_{1} \exp \left(-C_{2} \lambda K^{2}\right)\left|\left\{x \in Q: M_{\alpha}(\log u, Q)(x)>\lambda\right\}\right|
$$

(b) $\left|\left\{x \in Q: A_{\alpha}^{2}(\log u, Q)(x)>K \lambda, M_{\alpha}(\log u, Q)(x)<\lambda\right\}\right|$

$$
\leq C_{1} \exp \left(-C_{2} K \lambda\right)\left|\left\{x \in Q: A_{\alpha}^{2}(\log u, Q)(x)>\lambda\right\}\right|
$$

The proof follows the Bañuelos-Moore scheme of approximating a harmonic function by a dyadic martingale (see [6, Chapter 2]). In our case the function $\log u$ is not harmonic. However, the approximation scheme is flexible enough to deal with it. As in the martingale case, Theorem 1.5 stated in the Introduction will follow easily from the result above. However Theorem 1.5 (a) and therefore Corollary 1.6 can be proved in a more elementary way, as presented in the next paragraph.

\section{Characterization of singular measures}

Let $u$ be a positive harmonic function in the upper half space $\mathbb{R}_{+}^{N+1}$. Harnack's inequality states that there exists a constant $C=C(N)$ such that

$$
C^{-1} u(w, t) \leq u(x, y) \leq C u(w, t),
$$

for any pair of points $(x, y),(w, t) \in \mathbb{R}_{+}^{N+1}$ such that

$$
\|(w, t)-(x, y)\| \leq y / 2 .
$$

The infinitessimal version of Harnack's inequality is the following estimate

$$
\frac{y|\nabla u(x, y)|}{u(x, y)} \leq N
$$

which holds for any $(x, y) \in \mathbb{R}_{+}^{N+1}$. So, the function $\log u$ satisfies the Lipschitz condition

$$
|\log u(z)-\log u(w)| \leq C \rho(z, w),
$$

where $\rho(z, w)$ is the hyperbolic distance in $\mathbb{R}_{+}^{N+1}$. By Harnack's inequality and the submean property of gradient of $u$, we obtain

Lemma 3.2. Let $u$ be a positive harmonic function in $\mathbb{R}_{+}^{N+1}$. Let $B$ be a ball centered at $z \in \mathbb{R}_{+}^{N+1}$ such that $2 B \subset \mathbb{R}_{+}^{N+1}$. Then, there exists a constant $C=C(N)$ such that

$$
\left|\frac{\nabla u(z)}{u(z)}\right| \leq \frac{C}{|B|} \int_{B} \frac{|\nabla u(w)|}{u(w)} d m(w) .
$$


Recall that a positive harmonic function $u$ in $\mathbb{R}_{+}^{N+1}$ has a finite non-tangential limit at almost every point $x \in \mathbb{R}^{N}$. However, the limit may be 0 at a large set. The result we prove next is the following:

Theorem 3.3. Let $u$ be a positive harmonic function in the upper half space $\mathbb{R}_{+}^{N+1}$. Fix $\alpha>0$. Then, the sets

$\left\{x \in \mathbb{R}^{N}: \lim _{y \rightarrow 0} u(x, y)>0\right\} \quad$ and $\left\{x \in \mathbb{R}^{N}: \int_{\Gamma_{\alpha}(x)} \frac{|\nabla u(w, y)|^{2}}{u(w, y)^{2}} y^{1-N} d w d y<\infty\right\}$ can only differ in a set of Lebesgue measure 0.

As a consequence we get

Corollary 3.4. Let $\mu$ be a positive measure and let $u$ be its harmonic extension. Fix $\alpha>0$. Then $\mu$ is singular if and only if

$$
\int_{\Gamma_{\alpha}(x)} \frac{|\nabla u(w, y)|^{2}}{u(w, y)^{2}} y^{1-N} d w d y=\infty, \quad \text { a.e. } x \in \mathbb{R}^{N}
$$

Proof of Theorem 3.3. The proof is based on well known arguments using Green's formula on saw-tooth regions (see, for instance [35, Chap. VII]). Set

$$
A=\left\{x \in \mathbb{R}^{N}: \lim _{y \rightarrow 0} u(x, y)>0\right\} \quad \text { and } \quad B=\left\{x \in \mathbb{R}^{N}: A_{\alpha}(u)(x)<\infty\right\} .
$$

We first show that almost every point of $A$ is in $B$. So, let $E$ be a compact set and $\varepsilon>0$ such that

$$
\varepsilon^{-1}>\lim _{y \rightarrow 0} u(x, y)>\varepsilon
$$

Fix $\beta>\alpha$, Harnack's inequality provides constants $C=C(\beta, \varepsilon), K=K(\beta, \varepsilon)$ such that

$$
\inf _{(w, y) \in \Gamma_{\beta}(x)} u(w, y)>C, \quad \sup _{(w, y) \in \Gamma_{\beta}(x)} u(w, y)<K,
$$

for any $x \in E$. Renormalizing, we may assume

$$
\inf _{(w, y) \in \Gamma_{\beta}(x)} u(w, y)>\varepsilon, \quad \sup _{(w, y) \in \Gamma_{\beta}(x)} u(w, y)<\varepsilon^{-1},
$$

for any $x \in E$. Now, let

$$
R=\cup_{x \in E} \Gamma_{\alpha}(x)
$$

and note that $\varepsilon^{-1}>u>\varepsilon$ on $R$. Fubini's theorem gives

$$
\int_{E} A_{\alpha}^{2}(\log u)(x) d x \leq C \int_{R} y \frac{|\nabla u(x, y)|^{2}}{u(x, y)^{2}} d x d y .
$$


Since $\Delta(\log u)=-|\nabla u|^{2} / u^{2}$, Green's formula applied to the functions $\log u$ and $y$ on the domain $R$ yields

$$
\int_{R} y \frac{|\nabla u(x, y)|^{2}}{u(x, y)^{2}} d x d y=\int_{\partial R}(\log u) \partial_{\vec{n}} y-\int_{\partial R} y \partial_{\vec{n}}(\log u) .
$$

The first integral on the right side is bounded by $|\log \varepsilon||\partial R|$ and the second one by $N|\partial R|$ because by Harnack's inequality $y|\nabla(\log u)(x, y)| \leq N$. Hence,

and the proof is finished.

$$
\int_{E} A_{\alpha}^{2}(\log u)(x) d x<\infty
$$

Now, we want to show that almost every point of $B$ is in $A$. We argue by contradiction. So, we may assume

$$
\left|\left\{x \in \mathbb{R}^{N}: A_{\alpha}^{2}(\log u)(x) \leq 1, \lim _{y \rightarrow 0} u(x, y)=0\right\}\right|>0 .
$$

Then one may find a compact set $E$ of positive measure such that $u$ is non-tangentially bounded on $E$ and

$$
A_{\alpha}^{2}(\log u)(x) \leq 1, \quad \lim _{y \rightarrow 0} u(x, y)=0,
$$

for any $x \in E$. Observe that Harnack's inequality implies that

$$
u(w, t) \rightarrow 0,
$$

whenever $(w, t)$ tends non-tangentially to a point $x \in E$. Considering a subset of $E$ if necessary, one can assume that $\log u$ is bounded from above on the set

$$
R=\cup_{x \in E} \Gamma_{\alpha}(x) .
$$

Now applying Green's formula as above we get

$$
\int_{R} y \frac{|\nabla u(x, y)|^{2}}{u(x, y)^{2}} d x d y=\int_{\partial R}(\log u) \partial_{\vec{n}} y-\int_{\partial R} y \partial_{\vec{n}}(\log u) .
$$

By Fubini, the volume integral is bounded by a multiple of

$$
\int_{E} A_{\alpha}^{2}(\log u)(x) d x \leq|E|
$$

and by Harnack's inequality

$$
\left|\int_{\partial R} y \partial_{\vec{n}}(\log u)\right| \leq N|\partial R|
$$

However, since $\partial_{\vec{n}} y \geq 1 / 2$ at $\partial R \cap\{y=1\}$ and $\log u$ is bounded from above, we deduce

$$
\int_{\partial R}(\log u) \partial_{\vec{n}} y=-\infty
$$

which gives the contradiction. 
Last proof combined with stopping time arguments give versions of the estimates in Theorem 3.1 with constants having worst decay. Next we present a proof of the good- $\lambda$-inequalities stated in that Theorem. As explained before, the idea is to adapt the Bañuelos-Moore method to reduce the result to the martingale setting.

\section{Approximating $\log u$ by a dyadic martingale.}

The idea of approximating a function by a dyadic martingale using Calderón reproducing formula goes back to Chang, Wilson and Wolff [14]. Later, Bañuelos and Moore [6] related a harmonic function in the upper half space to a truncated version of a Calderón-type integral formula. Let us explain briefly their idea.

Let $u$ be a harmonic function in $\mathbb{R}_{+}^{N+1}$. Given $\rho>0$, fix a positive, smooth, radial function $K$ supported on $B(0, \rho) \subset \mathbb{R}^{N}$, with integral 1 . Then write

$$
\begin{aligned}
P(x) & =-\sum_{i=1}^{N} x_{i} \frac{\partial K}{\partial x_{i}}(x)-(N-1) K(x), \\
q(x) & =\left(-x_{1} P(x)+\frac{\partial K}{\partial x_{1}}(x), \ldots,-x_{N} P(x)+\frac{\partial K}{\partial x_{N}}(x)\right) .
\end{aligned}
$$

Finally write $q_{t}(x)=t^{-N} q(x / t)$ and a similar definition for $K_{t}(x)$. Define

$$
v(x, t)=\int_{\mathbb{R}^{N}} P_{t}(x-y) u(y, t) d y .
$$

The Bañuelos-Moore approach may be divided into the following steps.

Let $\varepsilon_{m}=2^{-m-2} / 4 \rho$ and recall that $A_{\alpha}(u)(x, t)$ denotes the truncated area function of $u$.

(I) Given $\alpha>0$, one can choose $\rho$ sufficiently small such that for $m=$ $1,2, \ldots$ if $\varepsilon_{m+1}<t_{0} \leq \varepsilon_{m}$,

$$
\left|u\left(x, t_{0}\right)-v\left(x, \varepsilon_{m}\right)\right| \leq C A_{\alpha}(u)\left(x, \gamma t_{0}\right),
$$

where $0<\gamma<1$ is a constant depending on $\rho$. See [6, p. 72].

(II) For $0<\varepsilon<\widetilde{\varepsilon} \leq 1 / 4$,

$$
v(x, \widetilde{\varepsilon})-v(x, \varepsilon)=\int_{\varepsilon}^{\widetilde{\varepsilon}} \int_{\mathbb{R}^{N}} q_{t}(x-w) \nabla_{w} u(w, t) d w d t+A(x)
$$

where

$$
A(x)=\int_{\mathbb{R}^{N}} \widetilde{\varepsilon} K_{\widetilde{\varepsilon}}(x-w) \frac{\partial u}{\partial t}(w, \widetilde{\varepsilon}) d w-\int_{\mathbb{R}^{N}} \varepsilon K_{\varepsilon}(x-w) \frac{\partial u}{\partial t}(w, \varepsilon) d w .
$$

Moreover, if $\rho$ is chosen sufficiently small with respect to $\alpha, A(x)$ is bounded by a fixed multiple of $A_{\alpha}(u)(x, \gamma \varepsilon)$. Here $0<\gamma<1$ is a constant depending on $\rho$. See $[6$, p. 56, p. 70]. 
(III) Consider the function

$$
V\left(x, \varepsilon_{m}\right)=\int_{\varepsilon_{m}}^{1 / 4} \int_{\mathbb{R}^{N}} q_{t}(x-w) \nabla_{w} u(w, t) d w d t .
$$

If $\rho$ is taken sufficiently small with respect to $\alpha$, there exist a positive number $L=L(N)$ and martingales (with respect to different translates of the usual dyadic filtration), $\left\{S_{n}^{(1)}\right\}, \ldots,\left\{S_{n}^{(L)}\right\}$, such that

$$
\begin{gathered}
\left|V\left(x, \varepsilon_{m}\right)-\sum_{k=1}^{L} S_{m}^{(k)}(x)\right| \leq C+C A_{\alpha}(u)\left(x, \gamma \varepsilon_{m}\right), \\
\sum_{k=1}^{L}\left\langle S^{(k)}\right\rangle_{m}(x) \leq C A_{\alpha}(u)\left(x, \gamma \varepsilon_{m}\right) .
\end{gathered}
$$

See $[6$, p. 50].

So, if $\rho$ is taken sufficiently small, combining (I), (II) and (III) we get that for $m=1,2, \ldots$ and $\varepsilon_{m+1}<t<\varepsilon_{m}$,

$$
\begin{gathered}
\left|u(x, t)-\sum_{k=1}^{L} S_{m}^{k}(x)\right| \leq C+C A_{\alpha}(u)(x, \gamma t), \\
\sum_{k=1}^{L}\left\langle S^{k}\right\rangle_{m}(x) \leq C A_{\alpha}(u)(x, \gamma t),
\end{gathered}
$$

where $\gamma$ is a fixed constant. These estimates allow us to transfer problems on the growth of the harmonic function $u$ on regions where its (truncated) area function is bounded to the martingale setting, that is, to problems on growth of martingales on regions where its quadratic variation remains bounded, since in this context the subgaussian estimate is available. In this scheme, the harmonicity of $u$ is used in different ways. The steps (I) and (III) only depend on the submean property of $|\nabla u|$, that is,

$$
\left|\nabla u\left(x_{0}, y_{0}\right)\right| \leq \frac{1}{|B|} \int_{B}|\nabla u(x, y)| d x d y
$$

where $B$ is a ball centered at $\left(x_{0}, y_{0}\right)$ contained in $\mathbb{R}_{+}^{N+1}$. However the identity in (II) uses the whole information $\Delta u=0$.

Now, given a positive harmonic function $u$, our strategy consists on replacing $u$ by $\log u$ in the previous scheme. So we consider

$$
v_{*}(x, t)=\int_{\mathbb{R}^{N}} P_{t}(x-w) \log u(w, t) d w .
$$


Recall that by Lemma 3.2 the submean value property for $|\nabla(\log u)|$ holds. So the proof of (I) and (III) by Bañuelos and Moore applies in our situation, that is,

$\left(\mathrm{I}^{*}\right)$ Given $\alpha>0$, one can choose $\rho$ sufficiently small such that for $m=$ $1,2, \ldots$ if $\varepsilon_{m+1}<t_{0} \leq \varepsilon_{m}$

$$
\left|\log u\left(x, t_{0}\right)-v_{*}\left(x, \varepsilon_{m}\right)\right| \leq C A_{\alpha}(\log u)\left(x, \gamma t_{0}\right) .
$$

(III*) Consider the function

$$
V_{*}\left(x, \varepsilon_{m}\right)=\int_{\varepsilon_{m}}^{1 / 4} \int_{\mathbb{R}^{N}} q_{t}(x-w) \nabla_{w} \log u(w, t) d w d t .
$$

If $\rho$ is chosen sufficiently small with respect to $\alpha$, there exists a positive number $L=L(N)$ and martingales (with respect to different translates of the usual dyadic filtration), $\left\{S_{n}^{(1)}\right\}, \ldots,\left\{S_{n}^{(L)}\right\}$ such that

$$
\begin{aligned}
& \mid V_{*}\left(x, \varepsilon_{m}\right)- \sum_{k=1}^{L} S_{m}^{(k)}(x) \mid \leq C+C A_{\alpha}(\log u)\left(x, \gamma \varepsilon_{m}\right), \\
& \sum_{k=1}^{L}\left\langle S^{k}\right\rangle_{m}(x) \leq C A_{\alpha}(\log u)\left(x, \gamma \varepsilon_{m}\right) .
\end{aligned}
$$

The analogue of (II) for $\log u$ is harder to establish and it is the main content of the two following results.

Lemma 3.5. Let $u$ be a positive harmonic function in $\mathbb{R}_{+}^{N+1}$. Given a positive, smooth, radial function $K$ supported on $\left\{x \in \mathbb{R}^{N}:\|x\|<\rho\right\}$, with integral 1, consider the functions $P, q$ given in (3.1) and (3.2). Define

$$
v_{*}(x, t)=\int_{\mathbb{R}^{N}} P_{t}(x-w) \log u(w, t) d w .
$$

Then, for $0<\varepsilon<1 / 4$,

$$
v_{*}(x, 1 / 4)-v_{*}(x, \varepsilon)=V_{*}(x, \varepsilon)+Q(x, \varepsilon)+R(x, \varepsilon),
$$

where

$$
\begin{aligned}
V_{*}(x, \varepsilon)= & \int_{\varepsilon}^{1 / 4} \int_{\mathbb{R}^{N}} q_{t}(x-w) \nabla_{w}(\log u)(w, t) d w d t, \\
Q(x, \varepsilon)= & \int_{\varepsilon}^{1 / 4} \int_{\mathbb{R}^{N}} t K_{t}(x-w) \frac{|\nabla u(w, t)|^{2}}{u(w, t)^{2}} d w d t, \\
R(x, \varepsilon)= & \int_{\mathbb{R}^{N}} \frac{1}{4} K_{1 / 4}(x-w) \frac{\partial \log u}{\partial t}(w, 1 / 4) d w \\
& -\int_{\mathbb{R}^{N}} \varepsilon K_{\varepsilon}(x-w) \frac{\partial \log u}{\partial t}(w, \varepsilon) d w .
\end{aligned}
$$


Proof. We follow the proof of (II) by Bañuelos and Moore (see [6, p. 56]). Let $0<\varepsilon<\widetilde{\varepsilon}$. Applying the fundamental Theorem of Calculus, we get

$$
\begin{aligned}
v_{*}(x, \widetilde{\varepsilon})-v_{*}(x, \varepsilon)= & \left.\int_{\varepsilon}^{\widetilde{\varepsilon}} \int_{\mathbb{R}^{N}} \frac{\partial}{\partial t}\left(t^{-N} P((x-w) / t)\right)\right) \log u(w, t) d w d t \\
& +\int_{\varepsilon}^{\widetilde{\varepsilon}} \int_{\mathbb{R}^{N}} t^{-N} P((x-w) / t) \partial_{t}(\log u)(w, t) d w d t .
\end{aligned}
$$

Using that

$$
\frac{\partial}{\partial t}\left(t^{-N} P((x-w) / t)\right)=\sum_{i=1}^{N} \frac{\partial}{\partial w_{i}}\left(\frac{x_{i}-w_{i}}{t^{N+1}} P((x-w) / t)\right)
$$

and integrating by parts, the first integral becomes

$$
-\int_{\varepsilon}^{\widetilde{\varepsilon}} \int_{\mathbb{R}^{N}} \sum_{i=1}^{N} \frac{x_{i}-w_{i}}{t^{N+1}} P((x-w) / t) \frac{\partial \log u}{\partial w_{i}}(w, t) d w d t .
$$

Using that

$$
t^{-N} P((x-w) / t)=\frac{\partial}{\partial t}\left(t^{-N+1} K((x-w) / t)\right),
$$

and an integration by parts, the second integral becomes

$$
\begin{aligned}
& -\int_{\varepsilon}^{\widetilde{\varepsilon}} \int_{\mathbb{R}^{N}} t^{-N+1} K((x-w) / t) \frac{\partial^{2}}{\partial t^{2}}(\log u)(w, t) d w d t \\
& +\int_{\mathbb{R}^{N}} \widetilde{\varepsilon} K_{\widetilde{\varepsilon}}(x-w) \frac{\partial \log u}{\partial t}(w, \widetilde{\varepsilon}) d w-\int_{\mathbb{R}^{n}} \varepsilon K_{\varepsilon}(x-w) \frac{\partial \log u}{\partial t}(w, \varepsilon) d w \\
= & A+B+C .
\end{aligned}
$$

Since

$$
\frac{\partial^{2} \log u}{\partial t^{2}}=-\sum_{i=1}^{N} \frac{\partial^{2} \log u}{\partial w_{i}^{2}}-\frac{|\nabla u|^{2}}{u^{2}}
$$

we get

$$
\begin{aligned}
A= & \int_{\varepsilon}^{\widetilde{\varepsilon}} \int_{\mathbb{R}^{N}} t^{-N+1} K((x-w) / t) \sum_{i=1}^{N} \frac{\partial^{2} \log u}{\partial w_{i}^{2}}(w, t) d w d t \\
& +\int_{\varepsilon}^{\widetilde{\varepsilon}} \int_{\mathbb{R}^{N}} t^{-N+1} K((x-w) / t) \frac{|\nabla u(w, t)|^{2}}{u(w, t)^{2}} d w d t
\end{aligned}
$$

Another integration by parts shows that the first term can be written as

$$
-\int_{\varepsilon}^{\widetilde{\varepsilon}} \int_{\mathbb{R}^{N}} t^{-N+1} \sum_{i=1}^{N}\left(\frac{\partial K}{\partial w_{i}}((x-w) / t)\right)\left(\frac{-1}{t} \frac{\partial \log u}{\partial w_{i}}(w, t)\right) d w d t .
$$


So, collecting all the identities, one gets

$$
\begin{aligned}
v(x, \widetilde{\varepsilon})-v(x, \varepsilon)= & \int_{\varepsilon}^{\widetilde{\varepsilon}} \int_{\mathbb{R}^{N}} q_{t}(x-w) \nabla_{w}(\log u)(w, t) d w \\
& +\int_{\varepsilon}^{\widetilde{\varepsilon}} \int_{\mathbb{R}^{N}} t^{-N+1} K((x-w) / t) \frac{|\nabla u(w, t)|^{2}}{u(w, t)^{2}} d w d t \\
& +\int_{\mathbb{R}^{N}} \widetilde{\varepsilon} K_{\widetilde{\varepsilon}}(x-w) \frac{\partial \log u}{\partial t}(w, \widetilde{\varepsilon}) d w \\
& -\int_{\mathbb{R}^{N}} \varepsilon K_{\varepsilon}(x-w) \frac{\partial \log u}{\partial t}(w, \varepsilon) d w .
\end{aligned}
$$

This finishes the proof.

Theorem 3.6. Let $u$ be a positive harmonic function on $\mathbb{R}_{+}^{N+1}$. Fix $\alpha>0$. Let $K$ be a smooth, positive, radial function supported on $\left\{x \in \mathbb{R}^{N}:\|x\|<\rho\right\}$ with integral 1. Consider the function

$$
Q(x, \varepsilon)=\int_{\varepsilon}^{1 / 4} \int_{\mathbb{R}^{N}} t K_{t}(x-w) \frac{|\nabla u(w, t)|^{2}}{u(w, t)^{2}} d w d t .
$$

If $\rho / \alpha$ is small enough, there exists a positive number $L=L(N)$ and martingales (with respect to different translates of the dyadic filtration)

$$
\left\{S_{n}^{(1)}\right\}, \ldots,\left\{S_{n}^{(L)}\right\}
$$

such that for $m=1,2, \ldots$, and $\varepsilon_{m+1}<y \leq \varepsilon_{m}$,

$$
\begin{aligned}
\mid \log u(x, y)+Q\left(x, \varepsilon_{m}\right)- & \sum_{k=1}^{L} S_{m}^{(k)}(x) \mid \leq C+C A_{\alpha}(\log u)(x, \gamma y), \\
\sum_{k=1}^{L}\left\langle S^{(k)}\right\rangle_{m}(x) & \leq A_{\alpha}(\log u)(x, \gamma y),
\end{aligned}
$$

where $\varepsilon_{m}=2^{-m-2} / 4 \rho$ and $0<\gamma<1$ is a constant depending on $\rho$. Here $C$ is a constant depending on $\alpha$ and $N$.

Proof. Observe that Harnack's inequality and (I)* assert that

$$
\left|\log u(x, y)-v_{*}\left(x, \varepsilon_{m}\right)\right| \leq C A_{\alpha}(\log u)\left(x, \gamma \varepsilon_{m}\right)+C .
$$

By Lemma 3.5,

$$
v_{*}(x, 1 / 4)-v_{*}\left(x, \varepsilon_{m}\right)=V_{*}\left(x, \varepsilon_{m}\right)+Q\left(x, \varepsilon_{m}\right)+R\left(x, \varepsilon_{m}\right) .
$$


Now, (III $)^{*}$ provides the martingales which approximate $V_{*}\left(x, \varepsilon_{m}\right)$. Hence, it only remains to show that

$$
R\left(x, \varepsilon_{m}\right) \leq C+C A_{\alpha}(\log u)\left(x, \gamma \varepsilon_{m}\right) .
$$

It is enough to prove

$$
\left|\int_{\mathbb{R}^{N}} \varepsilon K_{\varepsilon}(x-w) \frac{\partial \log u}{\partial t}(w, \varepsilon) d w\right| \leq C A_{\alpha}(\log u)(x, \gamma \varepsilon) .
$$

Observe that the integral is over all the $w$ contained in the ball centered at $x$ of radius $\rho \varepsilon$. By the submean property of the gradient

$$
\left|\frac{\partial \log u}{\partial t}(w, \varepsilon)\right| \leq\left(\frac{1}{|B(w)|} \int_{B(w)}\left|\frac{\nabla u}{u}\right|^{2}\right)^{1 / 2},
$$

where $B(w)$ is a ball centered at $(w, \varepsilon)$ of radius $\eta \varepsilon$. Now if $\eta$ and $\rho$ are sufficiently small, there exists $\delta<1$ such that

$$
B_{w} \subset B_{\varepsilon}=\left\{z \in \mathbb{R}_{+}^{N+1}:\|z-(x, \varepsilon)\|<\delta \varepsilon\right\} \subset \Gamma_{\alpha}(x)
$$

for any $w$ such that $\|w-x\|<\rho \varepsilon$. Then

$$
\left|\frac{\partial \log u}{\partial t}(w, \varepsilon)\right| \leq C\left(\frac{1}{\varepsilon^{N+1}} \int_{B_{\varepsilon}} \frac{|\nabla u|^{2}}{u^{2}}\right)^{1 / 2} .
$$

So,

$$
\begin{aligned}
\left|\int_{\mathbb{R}^{N}} \varepsilon K_{\varepsilon}(x-w) \frac{\partial \log u}{\partial t}(w, \varepsilon) d w\right| & \leq C\left(\int_{B_{\varepsilon}} \varepsilon^{-N+1} \frac{|\nabla u|^{2}}{u^{2}}\right)^{1 / 2} \\
& \leq A_{\alpha}(\log u)(x, \gamma \varepsilon)
\end{aligned}
$$

This finishes the proof.

Next we will show that given $0<\beta<\alpha$, one can choose $\rho$ in such a way that the resulting quantity $Q(x, \varepsilon)$ is, roughly speaking, intermediate between $A_{\alpha}^{2}(\log u)(x, \varepsilon)$ and $A_{\beta}^{2}(\log u)(x, \varepsilon)$.

Lemma 3.7. Let $u, K, Q$ be as in Theorem 3.6. Let $\left\{x \in \mathbb{R}^{N}:\|x\| \leq \rho\right\}$ be the closed support of $K$. Let $0<\beta<\alpha$.

(a) If $\rho<\alpha$,

$$
Q(x, \varepsilon) \leq C A_{\alpha}^{2}(\log u)(x, \varepsilon)
$$

(b) If $\rho>\beta$,

$$
Q(x, \varepsilon) \geq C^{-1} \int_{\Gamma_{\beta}(x, \varepsilon) \cap\{t<1 / 4\}} t^{-N+1} \frac{|\nabla u(w, t)|^{2}}{u(w, t)^{2}} d w d t .
$$

Here $C$ is a constant depending on the function $K, \alpha$ and $\beta$. 
Proof. Since $K$ is supported in $\left\{x \in \mathbb{R}^{N}:\|x\|<\rho\right\}$, the integral over $w$ in the definition of $Q(x, \varepsilon)$, is really over $w$ in the ball of center $x$ and radius $\rho t$. So, letting $C=\sup |K|$, if $\rho<\alpha, Q(x, \epsilon)$ is bounded by

$$
C \int_{\varepsilon}^{1 / 4} t^{-N+1} \int_{\|w-x\|<\rho t} \frac{|\nabla u(w, t)|^{2}}{u(w, t)^{2}} d w d t \leq C \int_{\Gamma_{\alpha}(x) \cap\{t \geq \varepsilon\}} t^{-N+1} \frac{|\nabla u(w, t)|^{2}}{u(w, t)^{2}} d w d t
$$

This gives the first estimate.

Given $\beta<\rho$, we choose $0<\delta<1$ such that

$$
\Gamma_{\beta}(x) \subset\{(w, t):\|w-x\|<(1-\delta) \rho t\} .
$$

Now let $m=\min \{K(x):\|x\|<\rho(1-\delta)\}$. Thus

$$
\begin{aligned}
Q(x, \varepsilon) & \geq m \int_{\varepsilon}^{1 / 4} t^{1-N} \int_{\{w:\|w-x\|<(1-\delta) \rho t\}} \frac{|\nabla u(w, t)|^{2}}{u(w, t)^{2}} d w d t \\
& \geq m \int_{\Gamma_{\beta}(x) \cap\{t \leq 1 / 4\}} t^{1-N} \frac{|\nabla u(w, t)|^{2}}{u(w, t)^{2}} d w d t .
\end{aligned}
$$

The sets $\left\{x \in \mathbb{R}^{N}: A_{\alpha}(\log u)(x)<\infty\right\}$ do not depend on $\alpha$, except for sets of Lebesgue measure 0 . In their complement, next result shows that the order of growth of $A_{\alpha}(\log u)(x, y)$ does not depend on $\alpha$.

Lemma 3.8. Let $u$ be a positive harmonic function in $\mathbb{R}_{+}^{N+1}$. Let $0<\beta<\alpha$. Then, there exists a constant $C=C(\alpha, \beta, N)>0$ such that

$$
\limsup _{t \rightarrow 0} \frac{A_{\alpha}(\log u)(x, t)}{A_{\beta}(\log u)(x, t)} \leq C,
$$

at almost every point $x \in\left\{x \in \mathbb{R}^{N}: A_{\alpha}(\log u)(x)=\infty\right\}$.

Proof. By contradiction, assume that for any positive constant $C$ there exists a compact set $F=F(C)$ of positive Lebesgue measure such that

$$
\begin{aligned}
& \limsup _{t \rightarrow 0} \frac{A_{\alpha}(\log u)(x, t)}{A_{\beta}(\log u)(x, t)}>C, \\
& \lim _{t \rightarrow 0} A_{\alpha}(\log u)(x, t)=\infty,
\end{aligned}
$$

for any $x \in F$. Considering a point of density of $F$, one can assume that $F$ is contained in a cube $Q$ and $|F \cap Q| \geq(1-\varepsilon)|Q|$. Thus, for arbitrarily large $\lambda>0$, one can find a set $F_{\lambda} \subset Q,\left|F_{\lambda}\right| \geq(1-2 \varepsilon)|Q|$, such that for any $x \in F_{\lambda}$ there exists $t=t(x, \lambda)>0$ for which $A_{\alpha}(\log u)(x, t)=\lambda$ and $A_{\beta}(\log u)(x, t)<C^{-1} \lambda$. 
Next we run a stopping time argument. Let $\left\{Q_{j}\right\}$ be the maximal dyadic subcubes of $Q$ for which there exists $x \in Q_{j}$ satisfying

$$
A_{\alpha}(\log u)\left(x, l\left(Q_{j}\right)\right) \geq \lambda
$$

Since $F_{\lambda} \subset \cup Q_{j}$, we have

$$
\sum\left|Q_{j}\right| \geq(1-2 \varepsilon)|Q|
$$

Observe that Harnack's inequality yields that whenever $|x-w|<y$,

$$
\left|A_{\alpha}(\log u)(x, y)-A_{\alpha}(\log u)(w, y)\right| \leq C(N, \alpha) .
$$

Thus, for any $x \in Q_{j}$ one has

$$
A_{\alpha}(\log u)\left(x, l\left(Q_{j}\right)\right) \geq \lambda-C(N, \alpha) .
$$

Given a cube $Q$ in $\mathbb{R}^{N}$ we denote by $\widehat{Q}$ the set $\{(x, y): x \in Q, 0<y<l(Q)\}$. Let $\Omega=\widehat{Q} \backslash \cup \widehat{Q}_{j}$. Then Fubini gives

$$
\begin{aligned}
\int_{\Omega} y \frac{|\nabla u(w, y)|^{2}}{u(w, y)^{2}} d w d y & \geq C(\alpha) \int_{Q}\left(\int_{\Gamma_{\alpha}(x) \cap \Omega} y^{1-N} \frac{|\nabla u(w, y)|^{2}}{u(w, y)^{2}} d w d y\right) d x \\
& \geq C_{1}(\alpha) \lambda^{2}(1-2 \varepsilon)|Q|
\end{aligned}
$$

On the other hand, by Fubini again

$$
\int_{\Omega} y \frac{|\nabla u(w, y)|^{2}}{u(w, y)^{2}} d w d y \leq C(\beta) \int_{Q}\left(\int_{\Gamma_{\beta}(x) \cap \Omega} y^{1-N} \frac{|\nabla u(w, y)|^{2}}{u(w, y)^{2}} d w d y\right) d x
$$

and applying Txebicheff's inequality one can find a set $G \subset Q,|G| \geq$ $C(\alpha, \beta)|Q|$ such that

$$
\int_{\Gamma_{\beta}(x) \cap \Omega} y^{1-N} \frac{|\nabla u(w, y)|^{2}}{u(w, y)^{2}} d w d y \geq C(\alpha, \beta) \lambda^{2} .
$$

Let $\mathcal{B}$ be the family of the cubes $\left\{Q_{j}\right\}$ for which

$$
A_{\beta}(\log u)\left(x, l\left(Q_{j}\right)\right)>2 C^{-1} \lambda
$$

for some $x \in Q_{j}$. Then

$$
\sum_{\mathcal{B}}\left|Q_{j}\right|<2 \varepsilon|Q|
$$

If $C$ is large enough this gives the contradiction and finishes the proof. 
We are now ready to prove Theorem 3.1

Proof of Theorem 3.1. Given a cube $Q$ of center $x_{Q}$ and sidelength $l(Q)$, let us denote $z_{Q}=\left(x_{Q}, l(Q)\right)$ and

$$
T(Q)=\left\{(x, y) \in \mathbb{R}_{+}^{N+1}: x \in Q, l(Q) / 2<y<l(Q)\right\} .
$$

To prove (a), we consider $\left\{Q_{j}\right\}$ the maximal dyadic subcubes of $Q$ such that

$$
\sup _{z \in T\left(Q_{j}\right)}\left|\log u(z)-\log u\left(z_{Q}\right)\right|>\lambda+C(\alpha),
$$

where $C(\alpha)$ is a constant depending on $\alpha$ (and independent on $\lambda$ ) to be chosen later. It follows from Harnack's inequality that if $C(\alpha)$ is chosen conveniently, then

$$
\left\{x \in Q: M_{\alpha}(\log u, Q)(x)>\lambda\right\} \supset \bigcup_{j} Q_{j} .
$$

Also, by maximality and Harnack's inequality, we have

$$
\left|\log u\left(z_{Q_{j}}\right)-\log u\left(z_{Q}\right)\right| \leq \lambda+C_{1}(\alpha),
$$

where $C_{1}(\alpha)$ is another positive constant depending on $\alpha$. Now, in each $Q_{j}$ we will use the martingales $S_{m}^{(i)}$ given in Theorem 3.6. Let

$$
S_{m}=\sum_{i=1}^{L}\left(S_{m}^{(i)}-S_{m}^{(i)}\left(Q_{j}\right)\right) \chi_{Q_{j}} .
$$

Then, the estimates in Theorem 3.6 and Lemma 3.7 give

$$
\begin{aligned}
\left\{x \in Q_{j}:\right. & \left.M_{\alpha}\left(\log u, Q_{j}\right)(x)>(K-1) \lambda, A_{\alpha}^{2}\left(\log u, Q_{j}\right)(x)<\lambda\right\} \\
& \subseteq\left\{x \in Q_{j}: \sup _{m}\left|S_{m}(x)\right|>(K-2 C) \lambda-C,\langle S\rangle^{2}(x)<C+\lambda C\right\} .
\end{aligned}
$$

The subgaussian estimate (1.1) applied to $S_{m}^{(i)}, i=1, \ldots, L$, implies that the measure of this set is bounded by

$$
C_{1} \exp \left(-C_{2} \lambda K^{2}\right)\left|Q_{j}\right|
$$

Adding up, we deduce that

$$
\begin{aligned}
\mid\{x \in Q: & \left.M_{\alpha}(\log u, Q)(x)>K \lambda, A_{\alpha}^{2}(\log u, Q)(x)<\varepsilon \lambda\right\} \mid \\
& \leq C_{1} \exp \left(-C_{2} K^{2} \lambda\right) \sum\left|Q_{j}\right| \\
& \leq C_{1} \exp \left(-C_{2} K^{2} \lambda\right)\left|\left\{x \in Q: M_{\alpha}(\log u, Q)(x)>\lambda\right\}\right|,
\end{aligned}
$$

which is (a). 
The proof of (b) is similar. Let $\left\{Q_{j}\right\}$ be the maximal dyadic subcubes of $Q$ such that

$$
A_{\alpha}^{2}(\log u, Q)\left(x, l\left(Q_{j}\right)\right) \geq \lambda+C_{1},
$$

for some $x \in Q_{j}$, where $C_{1}=C_{1}(\alpha)$ is a constant depending on $\alpha$ (and independent on $\lambda$ ) to be chosen later. It follows from Harnack's inequality and the maximality that if $C$ is chosen conveniently, then

$$
\lambda \leq A_{\alpha}^{2}(\log u, Q)\left(x, l\left(Q_{j}\right)\right) \leq \lambda+2 C_{1},
$$

for any $x \in Q_{j}$. So,

$$
\left\{x \in Q: A_{\alpha}^{2}(\log u, Q)(x)>\lambda\right\} \supset \cup_{j} Q_{j} .
$$

Now, in each $Q_{j}$ we will run a stopping time argument to find cubes where $A^{2}(\log u, Q)$ has increased $k \lambda$ units. More concretely, for $j=1,2, \ldots$, let $\left\{Q_{j, l}: l=1,2, \ldots\right\}$ be the maximal dyadic subcubes of $Q_{j}$ such that

$$
A_{\alpha}^{2}\left(\log u, Q_{j}\right)\left(x, l\left(Q_{j, l}\right)\right) \geq(k-1) \lambda-C_{2}
$$

for some $x \in Q_{j, l}$. By Harnack's inequality

$$
(k-1) \lambda-C_{3} \leq A_{\alpha}^{2}\left(\log u, Q_{j}\right)\left(x, l\left(Q_{j, l}\right)\right) \leq(k-1) \lambda+C_{3},
$$

for any $x \in Q_{j, l}$, where $C_{3}$ is a constant depending on $\alpha, C_{2}$ and the dimension. If the constants are chosen conveniently the set $\left\{x: A_{\alpha}^{2}(\log u, Q)(x)>k \lambda\right\}$ is contained in $\cup_{j, l} Q_{j, l}$. Let $A$ be the subfamily of $\left\{Q_{j, l}, j=1,2 \ldots, l=1,2 \ldots\right\}$ for which $M_{\alpha}(\log u, Q)(x) \leq \lambda$, for any $x \in Q_{j, l}$. Now in each $Q_{j}$ we will use the martingale $S_{m}^{(i)}$ given in Theorem 3.6. Let

$$
S_{m}=\sum_{i=1}^{L}\left(S_{m}^{(i)}-S_{m}^{(i)}\left(Q_{j}\right)\right) \chi_{Q_{j}}
$$

The estimates in Theorem 3.6 and Lemma 3.7 give that for any $Q_{j, l}$ in $A$, if $2^{-m-2} / 4 \rho=l\left(Q_{j, l}\right)$,

$$
\begin{aligned}
S_{m}(x) & \geq C_{4} k \lambda, \\
\langle S\rangle_{m}(x) & \leq C_{5} \sqrt{k \lambda},
\end{aligned}
$$

for any $x \in Q_{j, l}$. Hence, the subgaussian estimate (1.1) implies that

$$
\sum_{l}\left|Q_{j, l}\right| \leq C_{6} \exp \left(-C_{7} k \lambda\right)\left|Q_{j}\right|
$$

Adding up, we obtain (b). 
It is worth noticing that if one tries to adapt the proof of the corresponding subgaussian estimate for harmonic functions to our setting, a worst decay on the constants is obtained, as we show next. Let $\beta>\alpha>0$ and set

$$
E=\left\{x \in Q: M_{\alpha}(\log u, Q)(x)<\lambda\right\}
$$

and

$$
R=\bigcup_{x \in E} \Gamma_{\beta}(x) .
$$

Thus, $R$ is a Lipschitz domain say $R=\{(x, y): y>\phi(x)\}$, where $\phi$ is a Lipschitz function. Observe that

$$
|\log u(x, y)|<\lambda+C(\alpha, \beta),(x, y) \in R,
$$

where $C(\alpha, \beta)$ is a constant depending on $\alpha, \beta$.

Claim. Let

$$
A_{\alpha}^{2}(\log u)(x, \phi(x))=\int_{\Gamma_{R}((x, \phi(x)), \alpha)} \frac{|\nabla u(w, y)|^{2}}{u(w, y)^{2}}(y-\phi(x))^{1-N} d w d y,
$$

where $\Gamma_{R}((x, \phi(x)), \alpha)$ is a vertical cone with vertex at $(x, \phi(x))$ of aperture $\alpha$. Then

$$
\left\|A_{\alpha}^{2}(\log u)(x, \phi(x))\right\|_{\mathrm{BMO}(Q)} \leq C_{1} \lambda .
$$

This is the main step in Uchiyama's proof [38]. Then, the John-Niremberg Theorem implies

$$
\begin{aligned}
\mid\{x \in Q: & \left.A_{\alpha}^{2}(\log u, Q)(x)>K \lambda, M_{\alpha}(\log u, Q)(x)<\lambda\right\} \mid \\
& =\left|\left\{x \in Q: A_{\alpha}^{2}(\log u)(x, \phi(x))>K \lambda\right\}\right| \\
& \leq C_{1} \exp \left(-C_{2} K\right)\left|\left\{x \in Q: A_{\alpha}^{2}(\log u, Q)(x)>\lambda\right\}\right| .
\end{aligned}
$$

As in the martingale setting, the good- $\lambda$-inequalities have a number of consequences.

Theorem 3.9. Let $\mu$ be a positive measure on $\mathbb{R}^{N}$ such that

$$
\int_{\mathbb{R}^{N}} \frac{d \mu(x)}{1+|x|^{N+1}}<\infty
$$

Let $\mu=f d x+\mu_{s}$, where $f d x$ and $\mu_{s}$ are its absolutely continuous and singular part with respect to Lebesgue measure. Fix $\alpha>0$. Let $u=P[\mu]$ be the harmonic extension of $\mu$ and

$$
A_{\alpha}^{2}(\log u)(x)=\int_{\Gamma_{\alpha}(x)} \frac{|\nabla u(w, y)|^{2}}{u(w, y)^{2}} y^{1-N} d w d y
$$

its square function. Then, 
(a) The sets

$$
\left\{x \in \mathbb{R}^{N}: f(x)>0\right\} \quad \text { and } \quad\left\{x \in \mathbb{R}^{N}: A_{\alpha}^{2}(\log u)(x)<\infty\right\}
$$

can only differ in a set of Lebesgue measure 0.

(b) $\mu$ is a singular measure if and only if

$$
A_{\alpha}^{2}(\log u)(x)=\infty, \quad \text { a.e. } x \in \mathbb{R}^{N} .
$$

(c) Consider the non-tangential maximal function of $\log u$,

$$
M_{\alpha}(\log u)(x)=\sup _{\Gamma_{\alpha(x)}}|\log u| .
$$

Let $Q$ be a cube in $\mathbb{R}^{N}$. Then, for $0<p<\infty$ and any $\alpha>0$, $M_{\alpha}(\log u) \in L^{p}(Q)$ if and only if $A_{\alpha}^{2}(\log u) \in L^{p}(Q)$. Also, for $1<$ $p<\infty, \log f \in L^{p}(Q)$ if and only if $A_{\alpha}^{2}(\log u) \in L^{p}(Q)$.

(d) There exists a constant $C=C(N, \alpha)$ such that $\exp \left(C A_{\alpha}^{2}(\log u)\right) \in L_{\text {loc }}^{1}$ implies that $\mu$ and Lebesgue measure are mutually absolutely continuous.

(e) There exists a constant $c=c(N, \alpha)$ such that if $A_{\alpha}^{2}(\log u)(x)<1$ for any $x \in Q$, then

$$
\exp \left(c M_{\alpha}^{2}(\log u)\right) \in L^{1}(Q) .
$$

The proof is identical to the one in the dyadic martingale setting and we omit it.

\section{Law of the iterated logarithm}

Let $u$ be a positive harmonic function on $\mathbb{R}_{+}^{N+1}$. Given a positive, smooth, radial function $K$ supported on $B(0, \rho) \subset \mathbb{R}^{N}$, recall that $Q(x, \varepsilon)$ was defined as

$$
Q(x, \varepsilon)=\int_{\varepsilon}^{1 / 4} \int_{\mathbb{R}^{N}} t K_{t}(x-w)\left|\frac{\nabla u(w, t)}{u(w, t)}\right|^{2} d w d t .
$$

Observe that the integral is really over the $(w, t)$ in the cone $\Gamma_{\rho}(x, \epsilon)$ in $\mathbb{R}_{+}^{N+1}$. So, $Q(x, \epsilon)$ is a smooth version of the truncated area function $A_{\rho}^{2}(\log u)(x, \varepsilon)$. Hence, the sets

$$
\left\{x \in \mathbb{R}^{N}: \lim _{y \rightarrow 0} u(x, y)=0\right\} \quad \text { and } \quad\left\{x \in \mathbb{R}^{N}: \lim _{y \rightarrow 0} Q(x, y)=\infty\right\}
$$

can only differ in a set of Lebesgue measure 0 . 
Next result is an analogous of the LIL for dyadic measures given in Theorem 1.3.

Theorem 3.10. With the notations above

$$
\limsup _{y \rightarrow 0} \frac{|\log u(x, y)+Q(x, y)|}{\sqrt{Q(x, y) \log \log Q(x, y)}} \leq C
$$

at almost every point

$$
x \in\left\{x \in \mathbb{R}^{N}: \lim _{y \rightarrow 0} u(x, y)=0\right\}
$$

Here $C$ is a constant depending on $N$ and $K$.

Proof. We use Theorem 3.6 to get a collection of $L$ dyadic martingales $\left\{S_{m}^{(j)}\right\}_{j=1}^{L}$ such that

$$
\begin{aligned}
\mid \log u(x, y)+Q(x, y)- & \sum_{j=1}^{L} S_{m}^{(j)}(x) \mid \leq C+C A_{\alpha}(\log u)(x, \gamma y), \\
& \sum_{j=1}^{L}\left\langle S^{j}\right\rangle_{m}(x) \leq C A_{\alpha}(\log u)(x, \gamma y) .
\end{aligned}
$$

Here $m$ is chosen such that $\varepsilon_{m+1}<y \leq \varepsilon_{m}$, where $\varepsilon_{m}=2^{-m-2} / 4 \rho, 0<\gamma<1$ is a constant depending on $\rho$, and $\rho / \alpha$ is sufficiently small. Now, the LIL for martingales states

$$
\limsup _{m \rightarrow \infty} \frac{S_{m}^{(j)}(x)}{\sqrt{\left\langle S^{(j)}\right\rangle_{m}^{2}(x) \log \log \left\langle S^{(j)}\right\rangle_{m}(x)}} \leq C_{1},
$$

at a.e. $x \in\left\{x:\left\langle S^{(j)}\right\rangle(x)=\infty\right\}$. Hence, for any $j=1, \ldots, L$

$$
\limsup _{m \rightarrow \infty} \frac{S_{m}^{(j)}(x)}{\sqrt{A_{\alpha}^{2}\left(\log u, \varepsilon_{m}\right)(x) \log \log A_{\alpha}\left(\log u, \varepsilon_{m}\right)(x)}} \leq C_{1}
$$

for a.e. $x \in\left\{x \in \mathbb{R}^{N}: A_{\alpha}(\log u)(x)=\infty\right\}$. Then the result follows from (3.4) and Lemmas 3.7 and 3.8.

Applying the Theorem above and Lemmas 3.7 and 3.8, we deduce Corollary 1.8 . 


\section{An example}

There is a class of positive harmonic functions for which these LIL's give sharp results. Let $u$ be a positive harmonic function on $\mathbb{R}_{+}^{N+1}$. Harnack's inequality asserts

$$
y|\nabla u(x, y)| \leq N u(x, y), \quad(x, y) \in \mathbb{R}_{+}^{N+1} .
$$

Now given $\varepsilon>0$ and $R<1$, let $M(\varepsilon, R)$ be the class of positive harmonic functions $u$ for which

$$
\sup \left\{y \frac{|\nabla u(x, y)|}{u(x, y)}:\left\|(x, y)-\left(x_{0}, y_{0}\right)\right\| \leq R y_{0}\right\}>\varepsilon
$$

for any $\left(x_{0}, y_{0}\right) \in \mathbb{R}_{+}^{N+1}$. This is analogous to a class of holomorphic functions considered by P. Jones ([23]). For $\varepsilon>0$ small and $R$ close to 1 , the class $M(\varepsilon, R)$ is non trivial. For instance, if one considers the measure $\mu$ given in Proposition 2.9 and $u$ its Poisson extension, then $u \in M(\varepsilon, R)$ for some $\varepsilon$ and $R$. Now, if $u \in M(\varepsilon, R)$, the submean property given in Lemma 3.2, gives that

$$
Q(x, y) \geq C(\varepsilon, R) \log 1 / y,
$$

while Harnack's inequality gives

$$
Q(x, y) \leq C \log 1 / y .
$$

Similarly

$$
c(\alpha) \log 1 / y \leq A_{\alpha}^{2}(\log u)(x, y) \leq C(\alpha) \log 1 / y .
$$

So if $u \in M(\varepsilon, R)$, then $A_{\alpha}(\log u)(x)=\infty$ at almost every $x \in \mathbb{R}^{N}$, that is, $\lim _{y \rightarrow 0} u(x, y)=0$ a.e. $x \in \mathbb{R}^{N}$, or equivalently $u$ is the Poisson integral of a positive singular measure. Moreover, since

$$
\limsup _{y \rightarrow 0} \frac{|\log u(x, y)+Q(x, y)|}{\sqrt{\log 1 / y \log \log \log 1 / y}} \leq C, \quad \text { a.e. } x \in \mathbb{R}^{N},
$$

and

$$
C(\varepsilon, R) \log 1 / y \leq Q(x, y) \leq C \log 1 / y,
$$

we deduce that for a.e. $x \in \mathbb{R}^{N}$, one has

$$
\limsup _{y \rightarrow 0} \frac{|\log u(x, y)|}{\log 1 / y} \leq C
$$

and

$$
\liminf _{y \rightarrow 0} \frac{|\log u(x, y)|}{\log 1 / y}>C(\varepsilon, R) .
$$




\section{Hyperbolic derivatives of holomorphic mappings}

\section{Decay in Schwarz's lemma}

This subsection is devoted to the proof of Theorem 1.9. As explained in the introduction, (a) of Theorem 1.9 follows from Theorem 1.5 (a). To prove (b) it is more convenient to consider the upper half plane. An analytic function $I$ from the upper-half plane $\mathbb{R}_{+}^{2}$ to the unit disc is called inner if

$$
\lim _{y \rightarrow 0} I(x+i y)
$$

has modulus 1 at almost every $x \in \mathbb{R}$.

We apply Proposition 2.9 to obtain a positive, singular, doubling measure $\mu$ such that

$$
\left|\frac{\mu\left(I_{z}\right)}{\mu\left(I_{z}^{\prime}\right)}-1\right| \leq C h(z) \operatorname{Im} z, \quad 1 \geq \operatorname{Im} z>0,
$$

where $I_{z}$ is the interval of the real line centered at $\operatorname{Re}(z)$ of length $\operatorname{Im} z$. Let $u$ be the harmonic extension of $\mu$ to the upper half plane and $H=u+i \widetilde{u}$ where $\widetilde{u}$ is the unique harmonic function in $\mathbb{R}_{+}^{2}$ with $\widetilde{u}(i)=0$ such that $H=u+i \widetilde{u}$ is analytic in $\mathbb{R}_{+}^{2}$. Finally $I=\tau^{-1} \circ H$, where $\tau$ is a Möbius map from the unit disc to the right- half plane. Since $\mu$ is singular, the function $I$ is inner. Also,

$$
2 \frac{\left|I^{\prime}(z)\right|}{1-|I(z)|^{2}}=\frac{|\nabla u(z)|}{u(z)}, \quad \operatorname{Im} z>0 .
$$

So, we have to show that

$$
\frac{|\nabla u(z)|}{u(z)} \leq C h(z)
$$

for any $z=x+i y \in \mathbb{R}_{+}^{2}$. An integration by parts shows

$$
\frac{\partial u}{\partial y}(x, y)=-\frac{1}{2} \int_{\mathbb{R}} \frac{\partial^{2} P_{y}(t)}{\partial y \partial t} \operatorname{sign}(t)[\mu(x, x+t)-\mu(x-t, x)] d t
$$

and

$$
\frac{\partial u}{\partial x}(x, y)=\frac{1}{2} \int_{\mathbb{R}} \frac{\partial^{2} P_{y}(t)}{\partial t^{2}} \operatorname{sign}(t)[\mu(x, x+t)-\mu(x-t, x)] d t
$$

(see Lemma 2.2 in [16]). Here $P_{y}(t)=\pi^{-1} y\left(t^{2}+y^{2}\right)^{-1}$ is the Poisson kernel in the upper-half plane. We split these integrals dyadically, that is, over the intervals $I_{k} \backslash I_{k-1}, k=1,2, \ldots$, where $I_{k}$ is the interval associated to $x+i 2^{k} y$. Using the estimate

$$
\left|\frac{\partial^{2} P_{y}(t)}{\partial t^{2}}\right|+\left|\frac{\partial^{2} P_{y}(t)}{\partial y \partial t}\right| \leq \frac{C_{1}}{2^{4 k} y^{3}}, \quad \text { if }|t|<2^{k} y,
$$


and (4.1), one deduces

$$
\begin{aligned}
|\nabla u(x, y)| & \leq C_{2} \sum_{k=0}^{\infty} \frac{1}{2^{4 k} y^{3}} \mu\left(x, x+2^{k} y\right) h\left(x+i 2^{k} y\right)\left(2^{k} y\right)^{2} \\
& =C_{2} \sum_{k=0}^{\infty} \frac{1}{2^{2 k} y} \mu\left(x, x+2^{k} y\right) h\left(x+i 2^{k} y\right) .
\end{aligned}
$$

Since we are now working in the upper-half plane, the function $h(x+i y)$ is decreasing for $y \in(0,1 / 2]$. Therefore

$$
|\nabla u(x, y)| \leq C_{2} h(x+i y) \sum_{k=0}^{\infty} \frac{1}{2^{2 k} y} \mu\left(x, x+2^{k} y\right) \leq C_{3} h(x+i y) u(x, y),
$$

because $P_{y}(x-t)$ is comparable to $1 / 2^{2 k} y$ for $t \in I_{k} \backslash I_{k-1}$. This finishes the proof.

\section{Law of the iterated logarithm}

For $0 \leq \gamma<2 \pi$, let $\tau_{\gamma}(z)=\left(e^{i \gamma}+z\right)\left(e^{i \gamma}-z\right)^{-1}$ be a Möbius transformation from the unit disc onto the right-half plane. Given a holomorphic mapping $I$ from the unit disc into itself, let $u_{\gamma}$ be the real part of $\tau_{\gamma} \circ I$. Thus

$$
u_{\gamma}(z)=\frac{1-|I(z)|^{2}}{\left|e^{i \gamma}-I(z)\right|^{2}}, \quad z \in \mathbb{D}
$$

and a simple calculation shows that

$$
2 \frac{\left|I^{\prime}(z)\right|}{1-|I(z)|^{2}}=\frac{\left|\nabla u_{\gamma}(z)\right|}{u_{\gamma}(z)}, \quad z \in \mathbb{D},
$$

which is independent of $\gamma$. So, moving the notation from the upper-half plane to the disc, the truncated area function $\mathcal{A}_{\alpha}^{2}(I)\left(r e^{i \theta}\right)$ coincides with $4^{-1} A_{\alpha}^{2}\left(\log u_{\gamma}\right)\left(r e^{i \theta}\right)$ and $\mathcal{Q}\left(r e^{i \theta}\right)$ is $4^{-1} Q_{\alpha}\left(\log u_{\gamma}\right)\left(r e^{i \theta}\right)$. Thus by Theorem 1.7

$$
\limsup _{r \rightarrow 1} \frac{\left|\log \left(u_{\gamma}\left(r e^{i \theta}\right)\right)+\mathcal{Q}\left(r e^{i \theta}\right)\right|}{\sqrt{\mathcal{Q}\left(r e^{i \theta}\right) \log \log \mathcal{Q}\left(r e^{i \theta}\right)}} \leq C,
$$

Now, since for any $z \in \mathbb{D}$, one has

$$
\int_{o}^{2 \pi} \log \left|e^{i \gamma}-z\right|^{2} d \gamma=0
$$

Theorem 1.10 follows integrating on $\gamma$. 


\section{Inner functions in complex domains}

Let $\Omega$ be a hyperbolic domain in the complex plane, that is, $\Omega \subset \mathbb{C}$ such that $\mathbb{C} \backslash \Omega$ contains at least two points. Let $\lambda_{\mathbb{D}}(z)|d z|=\left(1-|z|^{2}\right)^{-1}|d z|$ be the Poincaré (or hyperbolic) metric in $\mathbb{D}$ and $\Pi: \mathbb{D} \rightarrow \Omega$ a holomorphic covering map onto $\Omega$. Then $\lambda_{\mathbb{D}}(z)$ projects to the Poincaré metric in the domain $\Omega, \lambda_{\Omega}(z)|d z|$, that is,

$$
\lambda_{\Omega}(\Pi(z))\left|\Pi^{\prime}(z)\right|=\lambda_{\mathbb{D}}(z), \quad z \in \mathbb{D} .
$$

Let $f$ be a holomorphic mapping from $\mathbb{D}$ into $\Omega$. Then, Schwarz's lemma asserts that

$$
\lambda_{\Omega}(f(z))\left|f^{\prime}(z)\right| \leq \lambda_{\mathbb{D}}(z), \quad z \in \mathbb{D},
$$

that is,

$$
\left(1-|z|^{2}\right)\left|f^{\prime}(z)\right| \lambda_{\Omega}(f(z)) \leq 1, \quad z \in \mathbb{D} .
$$

A holomorphic mapping $I$ from the unit disc into $\Omega$ is called inner into $\Omega$ if the set

$$
\left\{e^{i \theta} \in \partial \mathbb{D}: \lim _{r \rightarrow 1} I\left(r e^{i \theta}\right) \quad \text { exists and belongs to } \Omega\right\}
$$

has length 0. R. Nevanlinna proved that a holomorphic mapping from the unit disc into a domain whose complement has positive logarithmic capacity, has radial limit along almost every radius. (see [32, p. 209]). So, given such a domain $\Omega \subset \mathbb{C}$, a holomorphic mapping $I$ from $\mathbb{D}$ into $\Omega$ is inner into $\Omega$ if and only if

$$
\lim _{r \rightarrow 1} I\left(r e^{i \theta}\right) \in \partial \Omega
$$

for almost every $e^{i \theta} \in \partial \mathbb{D}$.

The functions which are inner into $\mathbb{D}$ are the usual inner functions. A covering map $\Pi$ from $\mathbb{D}$ onto a domain $\Omega$ is inner into $\Omega$ and, as a matter of fact, if $f$ is a holomorphic mapping from $\mathbb{D}$ into $\Omega$ which factorizes as $f=\Pi \circ b$, where $b: \mathbb{D} \rightarrow \mathbb{D}$, then $f$ is inner into $\Omega$ if and only if $b$ is an inner function (see [19]). Also,

$$
\lambda_{\Omega}(f(z))\left|f^{\prime}(z)\right|=\lambda_{\Omega}(\Pi(b(z)))\left|\Pi^{\prime}(b(z))\right|\left|b^{\prime}(z)\right|=\lambda_{\mathbb{D}}(b(z))\left|b^{\prime}(z)\right| .
$$

Thus, Theorem 1.9 gives the following result.

Corollary 4.1. Let $\Omega$ be a hyperbolic domain in the complex plane. Fix $\alpha>0$. Let $f$ be a holomorphic mapping from the unit disc into $\Omega$. Then $f$ is inner into $\Omega$ if and only if

$$
\int_{\Gamma_{\alpha}(\theta)} \lambda_{\Omega}^{2}(f(z))\left|f^{\prime}(z)\right|^{2} d m(z)=\infty
$$

for almost every $\theta \in[0,2 \pi]$. 


\section{Quasiconformal Mappings}

This section is devoted to the proof of Theorem 1.11. We start by proving some auxiliary results.

Lemma 5.1. With the notation of Theorem 1.11, the following identity holds:

$$
|\partial u(z)|=\left|\int_{\mathbb{R}_{-}^{2}} \frac{\bar{\partial} \rho(w)}{(w-z)^{3}} d m(w)\right|, \quad \text { for any } z \in \mathbb{R}_{+}^{2} .
$$

Proof. Since $u(z)=R e\left(i \int_{\mathbb{R}} \frac{d \mu(t)}{z-t}\right)$, one has

$$
|\partial u(z)|=\left|\partial_{z} \int_{\mathbb{R}} \frac{d \mu(t)}{z-t}\right|=\left|\int_{\mathbb{R}} \frac{d \mu(t)}{(z-t)^{2}}\right| .
$$

Approximating $\rho$ by smooth functions with compact support, we can assume that $d \mu(t)=\rho^{\prime}(t) d t$. Therefore the above integral is equal to

$$
\left|\int_{\mathbb{R}} \frac{\rho(t)}{(t-z)^{3}} d t\right|
$$

Now, Green's Theorem is applied to get

$$
|\partial u(z)|=\left|\int_{\mathbb{R}_{-}^{2}} \bar{\partial}_{w}\left(\frac{\rho(w)}{(w-z)^{3}}\right) d m(w)\right|=\left|\int_{\mathbb{R}_{-}^{2}} \frac{\bar{\partial} \rho(w)}{(w-z)^{3}} d m(w)\right| .
$$

Note that the lemma above holds under weaker assumptions: $\rho$ does not need to be quasiconformal.

Lemma 5.2. With the same notation as in Theorem 1.11,

$$
\frac{|\partial u(z)|}{u(z)} \leq \frac{C}{y^{1 / 2}}\left(\int_{y}^{\infty} \omega^{2}(z, t) \frac{d t}{t^{2}}\right)^{1 / 2}, \quad \text { for all } z=x+i y \in \mathbb{R}_{+}^{2},
$$

where

$$
\omega^{2}(z, t)=\frac{1}{t^{2}} \int_{B(z, t) \cap \mathbb{R}_{2}^{-}}|\sigma(w)|^{2} d m(w),
$$

and $B(z, t)$ denotes the ball centered at $z$ and radius $t$.

Proof. Since $\bar{\partial} \rho=\sigma \partial \rho$, by Lemma 5.1 we get

$$
|\partial u(z)| \leq \int_{\mathbb{R}_{2}^{-}} \frac{|\sigma(w)||\partial \rho(w)|}{|w-z|^{3}} d m(w) .
$$


Consider now the balls $B_{k}$ centered at $z$ of radius $2^{k} y$, and define

$$
D_{k}=B_{k} \cap \mathbb{R}_{2}^{-} \text {. }
$$

Denote by $J_{\rho}(w)$ the Jacobian of the quasiconformal mapping $\rho$, then

$$
\begin{aligned}
|\partial u(z)| & \lesssim \sum_{k=1}^{\infty} \frac{1}{2^{3 k} y^{3}} \int_{D_{k}}|\sigma(w)|\left|J_{\rho}(w)\right|^{1 / 2} d m(w) \\
& \cong \sum_{k=1}^{\infty} \frac{1}{2^{k} y} \int_{D_{k}}|\sigma(w)|\left|J_{\rho}(w)\right|^{1 / 2} d m(w) \\
& \leq \sum_{k=1}^{\infty} \frac{1}{2^{k} y}\left(f_{D_{k}}|\sigma(w)|^{2}\right)^{1 / 2}\left(f_{D_{k}}\left|J_{\rho}(w)\right|\right)^{1 / 2}
\end{aligned}
$$

where the symbol $f_{D} g$ represents the mean of the function $g$ over $D$. Now, by the circular distortion theorem the quantity Area $\left(\rho\left(D_{k}\right)\right)=\int_{D_{k}}\left|J_{\rho}(w)\right|$ is comparable to the square of the diameter of the interval $\rho\left(I_{k}\right)$, where $I_{k}$ is the interval centered at $x=R e z$ and length $2^{k} y$. Therefore

$$
|\partial u(z)| \lesssim \sum_{k=1}^{\infty} \frac{1}{2^{k} y}\left(f_{D_{k}}|\sigma(w)|^{2}\right)^{1 / 2} \frac{\mu\left(I_{k}\right)}{\left|I_{k}\right|},
$$

with all comparison constants depending only on the quasiconformal constant of the mapping $\rho$. Since $\mu$ is doubling, $\mu\left(I_{k}\right) \leq C^{k} \mu\left(I_{z}\right)$ where $I_{z}$ denotes the interval centered at $x=R e z$ and length $y$, and $C$ is the doubling constant. Writing $C=2^{1+\varepsilon}$, we get

$$
\frac{\mu\left(I_{k}\right)}{\left|I_{k}\right|} \leq 2^{k \varepsilon} \frac{\mu\left(I_{z}\right)}{\left|I_{z}\right|}
$$

Hence if $\varepsilon<1 / 2$

$$
\begin{aligned}
|\partial u(z)| & \leq \sum_{k} \frac{1}{2^{k} y}\left(f_{D_{k}}|\sigma(w)|^{2}\right)^{1 / 2} 2^{k \varepsilon} \frac{\mu\left(I_{z}\right)}{\left|I_{z}\right|} \\
& \leq u(z) \sum_{k} \frac{1}{2^{k(1-\varepsilon)} y}\left(f_{D_{k}}|\sigma(w)|^{2}\right)^{1 / 2} \\
& =\frac{u(z)}{y^{1 / 2}} \sum_{k} \frac{1}{2^{k(1 / 2-\varepsilon)}}\left(\frac{1}{2^{k} y} f_{D_{k}}|\sigma(w)|^{2}\right)^{1 / 2} \\
& \leq \frac{u(z)}{y^{1 / 2}}\left(\sum_{k} \frac{1}{2^{k(1-2 \varepsilon)}}\right)^{1 / 2}\left(\sum_{k} \frac{1}{2^{k} y} f_{D_{k}}|\sigma|^{2}\right)^{1 / 2} \\
& \lesssim K(C) \frac{u(z)}{y^{1 / 2}}\left(\int_{y}^{\infty} \omega^{2}(z, t) \frac{d t}{t^{2}}\right)^{1 / 2} .
\end{aligned}
$$


We finally proceed to prove Theorem 1.11.

Proof of Theorem 1.11. We have to show that

$$
\int_{\Gamma_{\alpha}(x)}\left(\frac{1}{y} \int_{y}^{\infty} \omega^{2}(z, t) \frac{d t}{t^{2}}\right) d m(z) \leq C \int_{\mathbb{R}_{2}^{-}} \frac{|\sigma(w)|^{2}}{|w-x|^{2}} d m(w) .
$$

By Fubini, the left term can be expressed as:

$$
\int_{\mathbb{R}_{2}^{-}}|\sigma(w)|^{2}\left(\int_{\Gamma_{\alpha}(x)} \frac{1}{y}\left(\int_{y}^{\infty} \frac{1}{t^{4}} \chi_{B(z, t)}(w) d t\right) d m(z)\right) d m(w) .
$$

On the other hand

$$
\int_{y}^{\infty} \frac{1}{t^{4}} \chi_{B(z, t)}(w) d t \simeq \int_{|z-w|}^{\infty} \frac{d t}{t^{4}} \simeq \frac{1}{|z-w|^{3}}
$$

and

$$
\int_{\Gamma_{\alpha}(x)} \frac{1}{y} \frac{d m(z)}{|z-w|^{3}} \lesssim \frac{1}{|x-w|^{2}}
$$

which ends the proof of the theorem.

\section{Concluding remarks}

\section{Centered square function}

Let $\mu$ be a doubling measure in $Q_{0}$. It is natural to consider analogues of the square function in Section 1 which do not use the dyadic filtration. Given a point $(w, y) \in \mathbb{R}_{+}^{N+1}$, let us consider

$$
d(w, y)=\max _{k}\left|\frac{2^{N} \mu\left(Q_{k}\right)}{\mu(Q)}-1\right|,
$$

where $Q$ is the cube in $\mathbb{R}^{N}$ centered at $w$ of sidelength $y$ and $\left\{Q_{k}: k=\right.$ $\left.1, \ldots, 2^{N}\right\}$ are the $2^{N}$ disjoint subcubes of $Q$ of sidelength $y / 2$. So, $d(w, y)$ measures the error done by $\mu$ when doubling at a cube centered at $w$ of sidelength $y$. Define

$$
\mathcal{A}_{\alpha}^{2}(\mu)(x)=\int_{\Gamma_{\alpha}(x)} d^{2}(w, y) \frac{d w d y}{y^{N+1}}, \quad x \in \mathbb{R}^{N} .
$$

Again, doubling measures which are singular can be characterized in terms of this square function. 
Theorem 6.1. Let $\mu$ be a doubling measure in $Q_{0}$. Fix $\alpha>0$. Then $\mu$ is singular if and only if

$$
\mathcal{A}_{\alpha}^{2}(\mu)(x)=\infty
$$

at almost every $x \in Q_{0}$.

Proof of Theorem 6.1. Given $0<\varepsilon<\eta$, let us consider the (doubly) truncated square function

$$
\mathcal{A}_{\alpha, \varepsilon, \eta}^{2}(\mu)(x)=\int_{\Gamma_{\alpha}(x) \cap\{\varepsilon<y<\eta\}} d^{2}(w, y) \frac{d w d y}{y^{N+1}} .
$$

The main step is to prove the following comparison estimate between integrals of the logarithm of the density of $\mu$ and the truncated square function $\mathcal{A}_{\alpha, \varepsilon, \eta}^{2}$. More concretely, there exists a constant $C=C(\mu, \alpha)>0$ such that for any cube $Q \subset Q_{0}$ and $0<y<l(Q) / 2$, one has

$$
\begin{aligned}
C^{-1}\left(1+\frac{1}{|Q|}\right. & \left.\int_{Q} \mathcal{A}_{\alpha, y, l(Q)}^{2}(\mu)(x) d x\right) \\
& \leq \frac{1}{|Q|} \int_{Q} \log \frac{\mu(Q(x, y))}{y^{N}} d x-\log \frac{\mu(Q)}{|Q|} \\
& \leq C\left(1+\frac{1}{|Q|} \int_{Q} \mathcal{A}_{\alpha, y, l(Q)}^{2}(\mu)(x) d x\right) .
\end{aligned}
$$

To show this, first note that, as in Lemma 2.2,

$$
d^{2}(w, y) \simeq-\log \frac{\text { g.m. }(Q(w, y))}{\text { a.m. }(Q(w, y))},
$$

where g.m. and a.m. are defined as in the martingale setting. By Fubini's Theorem

$$
\int_{Q} \mathcal{A}_{\alpha, y, l(Q)}^{2}(\mu)(x) d x \simeq-\int_{w \in Q} \int_{y}^{l(Q)} \log \frac{\text { g.m. }(Q(w, t))}{\operatorname{a.m} .(Q(w, t))} \frac{d t}{t} d w=\int_{y}^{l(Q)} \Phi(t) \frac{d t}{t},
$$

where

$$
\Phi(t)=-\int_{Q} \log \frac{\mathrm{g} \cdot \mathrm{m} \cdot(Q(w, t))}{\mathrm{a} \cdot \mathrm{m} \cdot(Q(w, t))} d w .
$$

Then, splitting the integral of $\Phi(t)$ dyadically and using the following cancellation property,

$$
\left|\int_{Q} \log \left(\frac{\text { g.m. } Q(w, t)}{\mu(Q(w, t / 2))}|Q(w, t / 2)|\right) d w\right| \leq C(\mu) t^{N}
$$

we obtain (6.1). Now Theorem 6.1 follows from standard stopping time arguments. We omit the details. 
Given a doubling measure $\mu$, let us consider the maximal function

$$
M_{\alpha}(\log \mu)(x)=\sup \left|\log \left(\frac{\mu(Q(w, y))}{y^{N}}\right)\right|,
$$

where the supremum is taken over the points $(w, y)$ in the cone $\Gamma_{\alpha}(x)$. Again, using (6.1), standard methods also give the analogue of part (b) in Theorem 1.1 , that is, for $0<p<\infty$,

$$
M_{\alpha}(\log \mu) \in L^{p}\left(Q_{0}\right) \quad \text { if and only if } \mathcal{A}_{\alpha}^{2}(\mu) \in L^{p}\left(Q_{0}\right) .
$$

However, we have not explored the analogues of (d) and (e) in Theorem 1.1 and the LIL of Theorem 1.3 for the square function $\mathcal{A}$. For this, it seems necessary to prove the analogue of the good $\lambda$-inequality, given in Theorem 2.4 (a).

\section{Sharp control of $A$ by $M$}

Theorem 2.4 and Theorem 3.1 establish comparisons between the distribution function of a non-tangential maximal function and a square function in two different settings: dyadic doubling measures and positive harmonic functions. Part (a) in both results provide the same subgaussian decay. However the estimate in part (b) in both results gives a worst decay and it probably could be improved. This is particularly convenient if one is interested on LIL's relating the growth of the (truncated) square function with the non-tangential maximal function. For harmonic functions, such LIL's were considered by Bañuelos and Moore (see [6, Chapter 4]).

\section{Non-harmonic extensions}

It is natural to consider the non-harmonic version of the results in Section 2. Let $\varphi: \mathbb{R}^{N} \rightarrow \mathbb{R}$ be a smooth function with integral 1 . Let $\mu$ be a positive measure on $\mathbb{R}^{N}$ such that

$$
\int_{\mathbb{R}^{N}}|\varphi(x)| d \mu(x)<\infty .
$$

Consider

$$
u(x, t)=\left(\varphi_{t} * d \mu\right)(x),
$$

where

$$
\varphi_{t}(x)=t^{-N} \varphi\left(\frac{x}{t}\right), \quad x \in \mathbb{R}^{N}, t>0 .
$$


Under some additional restrictions on the function $\varphi$, it is known that, for $0<p<\infty$, the non-tangential maximal function of $u$ is in $L^{p}$ if and only if the square function

$$
A_{\alpha}^{2}(\mu, \varphi)(x)=\int_{\Gamma_{\alpha}(x)}|\nabla u(w, t)|^{2} t^{1-N} d w d t
$$

is in $L^{p}$ (see [18]). In our setting, the natural square function to be considered is

$$
A_{\alpha}^{2}(\log \mu, \varphi)(x)=\int_{\Gamma_{\alpha}(x)}\left|\frac{\nabla u(w, t)}{u(w, t)}\right|^{2} t^{1-N} d w d t .
$$

We have not explored the square function in this generality. It is worth mentioning that in this generality, even the analogue of the subgaussian estimates of Chang, Wilson and Wolff is open (see [6, p. 113] and [36]). So, proving sharp estimates for $A^{2}(\log \mu, \varphi)$ seems to be a difficult problem.

One could also ask for results analogous to the ones in Section 2, with the area function replaced by the $g$-function

$$
g^{2}(\log u)(x)=\int_{0}^{1} y \frac{|\nabla u(x, y)|^{2}}{u(x, y)^{2}} d x .
$$

Again the main difficulty is the subgaussian estimate of Theorem 3.1, which is open in the context of harmonic functions (see [6, p. 114])

\section{References}

[1] Aleksandrov, A. B., Anderson, J. M. And Nicolau, A: Inner functions, Bloch spaces and symmetric measures. Proc. London Math. Soc. (3) 79 (1999), 318-352.

[2] Anderson, J. M., Fernández, J. L. and Shields, A. L.: Inner functions and cyclic vectors in the Bloch space. Trans. Amer. Math. Soc. 323 (1991), 429-448.

[3] Bañuelos, R., Klemeš, I. And Moore, C. N.: An analogue for harmonic functions of Kolmogorov's law of the iterated logarithm. Duke Math. J. 57 (1988), 37-68.

[4] Bañuelos, R., Klemeř, I. And Moore, C. N.: The lower bound in the law of the iterated logarithm for harmonic functions. Duke Math. J. 60 (1990), 689-715.

[5] Bañuelos, R. And Moore, C. N.: Laws of the iterated logarithm, sharp good- $\lambda$-inequalities and $L^{p}$-estimates for caloric and harmonic functions. Indiana Univ. Math. J. 38 (1989), 315-344. 
[6] Bañuelos, R. And Moore, C. N.: Probabilistic behavior of harmonic functions. Progress in Mathematics 175. Birkhäuser Verlag, Basel, 1999.

[7] Beurling, A. And Ahlfors, L. V.: The boundary correspondence under quasiconformal mappings. Acta Math. 96 (1956), 125-142.

[8] Brossard, J.: Intégrale d'aire et support d'une mesure positive. C. R. Acad. Sci. Paris Sér I. Math. 296 (1983), 231-232.

[9] Burkholder, D. L. ANd Gundy, R. F.: Extrapolation and interpolation of quasi-linear operators on martingales. Acta Math. 124 (1970), 249-304.

[10] Burkholder, D. L. And Gundy, R. F.: Distribution function inequalities for the area integral. Studia Math. 44 (1972), 527-544.

[11] BuCKLEy, S. M.: Estimates for operator norms on weighted spaces and reverse Jensen inequalities. Trans. Amer. Math. Soc. 340 (1993), 253-272.

[12] Cantón, A.: Singular measures and the little Bloch space. Publ. Mat. 42 (1998), 211-222.

[13] Carleson, L.: On mappings, conformal at the boundary. J. Analyse Math. 19 (1967), 1-13.

[14] Chang, S.-Y.A., Wilson, J. M. And Wolff, T. H.: Some weighted norm inequalities concerning the Schrödinger operator. Comment. Math. Helv. 60 (1985), 217-246.

[15] Dyn'kin, E.: Estimates for asymptotically conformal mappings. Ann. Acad. Sci. Fenn. Math. 22 (1997), 275-304.

[16] Doubtsov, E. And Nicolau, A.: Symmetric and Zygmund measures in several variables. Ann. Inst. Fourier (Grenoble) 52 (2002), no. 1, 153-177.

[17] Fefferman, R. A. Kenig, C. E. And Pipher, J.: The theory of weights and the Dirichlet problem for elliptic equations. Ann. of Math. (2) $\mathbf{1 3 4}$ (1991), 65-124.

[18] Fefferman, C. And Stein, E. M.: $H^{p}$ spaces of several variables. Acta Math. 129 (1972), 137-193.

[19] Fernández, J. L. And Nicolau, A.: Boundary behaviour of inner functions and holomorphic mappings. Math. Ann. 310 (1998), 423-445.

[20] Garnett, J. B. And Jones, P. W.: The distance in BMO to $L^{\infty}$. Ann. of Math. (2) 108 (1978), 373-393.

[21] Garnett, J. B. And Jones, P. W.: BMO from dyadic BMO. Pacific J. Math. 99 (1982), 351-371.

[22] Heurteaux, Y.: Sur la comparaison des mesures avec les mesures de Hausdorff. C. R. Acad. Sci. Paris Sér I Math. 321 (1995), 61-65. 
[23] Jones, P. W.: Square functions, Cauchy integrals, analytic capacity and harmonic measure. In Harmonic analysis and partial differential equations (El Escorial, 1987), 24-68. Lecture Notes in Math. 1384. Springer, Berlin, 1989.

[24] Kahane, J. P.: Trois notes sur les ensembles parfaits linéaires. Enseignement Math. (2) 15 (1969), 185-192.

[25] Kaufman, R. And Wu, J. M.: Two problems on doubling measures. Rev. Mat. Iberoamericana 11 (1995), no. 3, 527-545.

[26] Llorente, J. G.: Boundary values of harmonic Bloch functions in Lipschitz domains: a martingale approach. Potential Anal. 9 (1998), no. 3, 229-260.

[27] Makarov, N. G.: Probability methods in the theory of conformal mappings. (Russian) Algebra i Analiz 1 (1989), no. 1, 3-59. Translation in Leningrad Math. J. 1 (1990), no. 1, 1-56.

[28] Makarov, N. G.: Smooth measures and the law of the iterated logarithm. (Russian) Izv. Akad. Nauk SSSR Ser. Mat. 53 (1989), 439-446. Translation in Math. USSR-Izv. 34 (1990), 455-463.

[29] Macmanus, P.: Quasiconformal mappings and Ahlfors-David curves. Trans. Amer. Math. Soc. 343 (1994), 853-881.

[30] McConnell, T. R.: Area integrals and subharmonic functions. Indiana Univ. Math. J. 33 (1984), 289-303.

[31] Murai, T. And Uchiyama, A.: Good $\lambda$ inequalities for the area integral and the nontangential maximal function. Studia Math. 83 (1986), 251-262.

[32] Nevanlinna, R.: Analytic functions. Springer-Verlag, New York, Berlin, 1970.

[33] Piranian, G.: Two monotonic, singular, uniformly almost smooth functions. Duke Math. J. 33 (1966), 255-262.

[34] Smith, W.: Inner functions in the hyperbolic little Bloch class. Michigan Math. J. 45 (1998), 103-114.

[35] Stein, E. M.: Singular integrals and differentiability properties of functions. Princeton Mathematical Series 30. Princeton University Press, Princeton, N. J., 1970.

[36] Sweezy, C.: L-harmonic functions and the exponential square class. Pacific J. Math. 147 (1991), 187-200.

[37] Treil, S. R., Volberg, A. And Zheng, D.: Hilbert transform, Toeplitz operators and Hankel operators, and invariant $A_{\infty}$ weights. Rev. Mat. Iberoamericana 13 (1997), no. 2, 319-360.

[38] UChiyama, A.: On McConnell's inequality for functionals of subharmonic functions. Pacific J. Math. 128 (1987), 367-377. 
[39] Wu, J.-M.: Doubling measures with different basis. Colloq. Math. 76 (1998), no. 1, 49-55.

[40] Wu, J.-M.: Hausdorff dimension and doubling measures on metric spaces. Proc. Amer. Math. Soc. 126 (1998), no. 5, 1453-1459.

Recibido: 23 de mayo de 2002

\author{
María J. González \\ Departamento de Matemáticas (CASEM) \\ Universidad de Cádiz \\ 11510 Puerto Real, Cádiz (Spain) \\ majose.gonzalez@uca.es \\ Artur Nicolau \\ Departament de Matemàtiques \\ Facultat de Ciencies \\ Universitat Autònoma de Barcelona \\ 08193 Bellaterra, Barcelona (Spain) \\ artur@mat . uab.es
}

The authors are partially supported by the grants BFM2002-00571 and 2001SGR00431. 